DRAFT VERSION OCTOBER 25, 2018

Preprint typeset using LTEX style emulateapj v. 08/22/09

\title{
AN AMMONIA SPECTRAL ATLAS OF DENSE CORES IN PERSEUS
}

\author{
E. W. Rosolowsky ${ }^{1,2}$, J. E. Pineda ${ }^{2}$, J. B. Foster ${ }^{2}$, M. A. Borkin ${ }^{3}$, J. KAuffmann ${ }^{2,3}$, P. CAselli ${ }^{2,4}$, P. C. Myers ${ }^{2}$ And A. A. \\ GOODMAN 2,3 \\ Draft version October 25, 2018
}

\begin{abstract}
We present ammonia observations of 193 dense cores and core candidates in the Perseus molecular cloud made using the Robert F. Byrd Green Bank Telescope. We simultaneously observed the $\mathrm{NH}_{3}(1,1), \mathrm{NH}_{3}(2,2)$, $\mathrm{C}_{2} \mathrm{~S}\left(2_{1} \rightarrow 1_{0}\right)$ and $\mathrm{C}_{2}^{34} \mathrm{~S}\left(2_{1} \rightarrow 1_{0}\right)$ transitions near $\nu=23 \mathrm{GHz}$ for each of the targets with a spectral resolution of $\delta v \approx 0.024 \mathrm{~km} \mathrm{~s}^{-1}$. We find ammonia emission associated with nearly all of the (sub)millimeter sources as well as at several positions with no associated continuum emission. For each detection, we have measured physical properties by fitting a simple model to every spectral line simultaneously. Where appropriate, we have refined the model by accounting for low optical depths, multiple components along the line of sight and imperfect coupling to the GBT beam. For the cores in Perseus, we find a typical kinetic temperature of $T_{k}=11 \mathrm{~K}$, a typical column density of $N_{\mathrm{NH} 3} \approx 10^{14.5} \mathrm{~cm}^{-2}$ and velocity dispersions ranging from $\sigma_{v}=0.07 \mathrm{~km} \mathrm{~s}^{-1}$ to $0.7 \mathrm{~km} \mathrm{~s}^{-1}$. However, many cores with $\sigma_{v}>0.2 \mathrm{~km} \mathrm{~s}^{-1}$ show evidence for multiple velocity components along the line of sight.
\end{abstract}

Subject headings: ISM:clouds — ISM:molecules — radio lines:ISM

\section{INTRODUCTION}

Ammonia remains one of the best molecules for studying the cool, dense molecular cores where most stars form. The utility of ammonia was recognized early in the pursuit of molecular line astronomy (Ho \& Townes 1983, and references therein) and it remains the standard for identifying and studying the internal conditions of dense molecular cores. The unique quantum structure of the molecule coupled with its relative abundance allows for a host of measurements to be made from the hyperfine transitions among the multiple metastable states, which emit near $\nu=23 \mathrm{GHz}$. A single spectrum can be used to determine the line-of-sight velocity, velocity dispersion and gas kinetic temperature for a dense core. This set of properties form an excellent complement to surveys of submillimeter emission (e.g. Motte et al. 1998; Testi \& Sargent 1998) which readily study the size and distribution of the dust emission in cores, while yielding no information about the kinematics and temperatures.

Recently, submillimeter surveys of dense cores have been extended to cover large fractions of molecular clouds (Hatchell et al. 2005; Enoch et al. 2006), making complete surveys of dense cores possible. In addition, large scale mapping projects have surveyed several nearby molecular clouds at high resolution in emission from the isotopomers of CO (e.g., the COMPLETE Surveys of Serpens, Ophiuchus, Perseus; Ridge et al. 2006). The CO surveys establish the cores in the larger context of the molecular cloud. However, these surveys have raised many questions about the properties of cores and their relationship to the larger molecular environment.

To measure the kinetic temperature and kinematics of an

\footnotetext{
Electronic address: erosolow@cfa.harvard.edu

${ }^{1}$ National Science Foundation Astronomy and Astrophysics Postdoctoral Fellow

${ }^{2}$ Center for Astrophysics, 60 Garden St, Cambridge, MA 02138

${ }^{3}$ Initiative for Innovative Computing, Harvard University, 60 Oxford St., Cambridge, MA 02138

${ }^{4}$ School of Physics and Astronomy, University of Leeds, Leeds LS2 9JT,
}

unbiased sample of dense cores embedded in the same molecular complex, we have conducted a survey of dense cores in ammonia across the Perseus molecular cloud using the Green Bank Telescope. There are several advantages to adopting Perseus as a target. It has been extensively studied in several observational campaigns: the COMPLETE survey of star forming regions which surveyed the molecular gas using the FCRAO 14-m (Ridge et al. 2006); the SCUBA survey of submillimeter emission (Hatchell et al. 2005; Kirk et al. 2006); the BOLOCAM survey of the region in the $1.1 \mathrm{~mm}$ continuum (Enoch et al. 2006); and in all the Spitzer bands by the c2d project (Jørgensen et al. 2006; Rebull et al. 2007). The locations of dense cores have been identified in the (sub)millimeter maps, and their protostellar content has been explored (Jørgensen et al. 2007; Hatchell et al. 2007). In addition, Perseus shows a wide range of star forming environments, ranging from the newly formed clusters IC 348 and NGC 1333 to more isolated star forming regions such as B5 and L1448. A substantial portion of the molecular mass in the cloud is not currently forming stars.

Several previous observational studies provide context for the observations of Perseus. The observational results are homogenized in Jijina et al. (1999). The typical cores in Perseus have $\log \left(\mathrm{NH}_{3} / \mathrm{cm}^{-2}\right)=14.5, R_{p c}=0.09 \mathrm{pc}$ (after rescaling to our preferred distance of $260 \mathrm{pc}$ ), velocity dispersion $\sigma_{v}=0.17 \mathrm{~km} \mathrm{~s}^{-1}, T_{k}=11 \mathrm{~K}$. However, these studies have primarily observed the well known star forming regions with less concern for objects in the sterile portions of the molecular cloud.

This survey presents observations of $\mathrm{NH}_{3}$ and $\mathrm{C}_{2} \mathrm{~S}$ emission from a variety of sources in Perseus including millimeterbright dense cores as well as otherwise unremarkable high column density features selected from far infrared emission. The two tracers present complimentary views of the chemical evolution of the cloud. $\mathrm{C}_{2} \mathrm{~S}$ is regarded as an "earlytime" tracer formed in the initial conversion of atomic to molecular gas and excited at high densities $\left(n_{c r} \sim 10^{4.5} \mathrm{~cm}^{-3}\right.$ Langer et al. 1995; Di Francesco et al. 2006). As carbon 
species are depleted, $\mathrm{C}_{2} \mathrm{~S}$ disappears. In contrast, $\mathrm{NH}_{3}$ is regarded as a late-time tracer like $\mathrm{N}_{2} \mathrm{H}^{+}$, with both species requiring the relatively slow formation of $\mathrm{N}_{2}$ as a precursor. The molecules are excited at similar densities as $\mathrm{C}_{2} \mathrm{~S}$ but should not appear in significant amounts until later in the protostellar collapse $\left(t \sim 10^{5.5}\right.$ yr Flower et al. 2006; Di Francesco et al. 2006). Tafalla et al. (2004) note that the abundance of $\mathrm{NH}_{3}$ varies by a factor of several across starless cores while $\mathrm{N}_{2} \mathrm{H}^{+}$ remains constant. Such variations can complicate using ammonia as a structural tracer, but the utility of having a direct temperature measurement from the $\mathrm{NH}_{3}$ is immense.

Our survey of dense cores in Perseus adopted the form of a spectral survey to maximize the number of cores we could sample in a limited amount of time. Even with a single spectrum, we are able to determine core kinematics, velocity dispersion, kinetic temperatures, and chemical abundances of ammonia and $\mathrm{C}_{2} \mathrm{~S}$. We also spent a significant amount of time surveying core candidates derived through a variety of methods to find ammonia emission from objects not bright in the submillimeter. In this paper, we present the results of our survey and derive physical parameters from the ammonia spectra. A detailed comparison of the core properties to other tracers will be presented elsewhere.

\section{OBSERVATIONS}

We observed 193 dense cores and core candidates in the Perseus Molecular Cloud using the 100-m Robert F. Byrd Green Bank Telescope (GBT). The observations were conducted from 2 October - 10 November 2006 in eight separate observing shifts spanning a total of 59 hours. For each target we conducted single-pointing, frequency-switched observations for 5-30 minutes depending on the source. We used the high-frequency $K$-band receiver and configured the spectrometer to observe $412.5-\mathrm{MHz}$ windows centered on the rest frequencies of $\mathrm{NH}_{3}(1,1)(23.6944955(1) \mathrm{GHz}$, Lovas \& Dragoset 2003), $\mathrm{NH}_{3}(2,2)$ (23.7226333(1) GHz, Lovas \& Dragoset 2003), CCS $\left(2_{1} \rightarrow 1_{0}\right)(22.344033(1) \mathrm{GHz}$, Yamamoto et al. 1990) and $\mathrm{CC}^{34} \mathrm{~S}\left(2_{1} \rightarrow 1_{0}\right)(21.930476(1)$ $\mathrm{GHz}$, Ohishi \& Kaifu 1998). The frequency uncertainties translate to errors of $1-10 \mathrm{~m} \mathrm{~s}^{-1}$ uncertainties in our velocity scale and, for high signal-to-noise lines, limit the accuracy to which we can centroid the velocity. The spectrometer produces 8192 lags across each window yielding $1.525 \mathrm{kHz}$ channel separation with $1.862 \mathrm{kHz}$ resolution $\left(0.024 \mathrm{~km} \mathrm{~s}^{-1}\right.$ at this frequency) since the lags in the spectrometer are uniformly weighted. The frequency switch was asymmetric with a shift of $\pm 2.0599365 \mathrm{MHz}$ around the center of the band, allowing the entire $\mathrm{NH}_{3}(1,1)$ complex to remain within the spectral window.

We updated the pointing model of the telescope with observations of the quasar $0336+3218$ every $45-90$ minutes, depending on the wind conditions. In nearly all instances, the corrections to the model were $<10^{\prime \prime}$ except in the worst wind conditions (the GBT beam at $23 \mathrm{GHz}$ is $31^{\prime \prime}$ or $0.04 \mathrm{pc}$ at the assumed 260 pc of Perseus; Cernis 1993). Since the typical dense core size is $\sim 0.08$ pc for Perseus (Jijina et al. 1999) pointing deviations should not confuse sources with the exception of the most densely clustered regions (IC 348, NGC 1333). Some of the complex velocity structure seen in the ammonia spectra almost certainly results from multiple sources along the line of sight ( $\$ 3.4)$. However, sources are chosen to be separated by $\geq 1$ GBT beam FWHM confusion due to overlapping beams should be negligible compared to confu- sion intrinsic to the sources on the sky.

We calibrated the data with injection of a noise signal periodically throughout the observations. Because of slow variations in the power output of the noise diodes and their coupling to the signal path, we measured the strength of the noise signal through observations of a source with known flux (the NRAO flux calibrator 3C84). We repeated the flux calibration observations during every observing run to detect any changes in the calibration sources, finding no significant variations over the course of our run. Calibrating the noise diodes established the $T_{A}$ scale, and we scaled to the $T_{A}^{*}$ scale using estimates of the atmospheric opacity at $22-23 \mathrm{GHz}$ from models of the atmosphere derived using weather data ${ }^{5}$. To reach the $T_{m b}$ scale, the spectra are divided by the main beam efficiency of the GBT, which is $\eta_{m b} \approx 0.81$ at these frequencies. We observed one of our sources (NH3SRC 47, where $\mathrm{NH} 3 \mathrm{SRC}$ is our source catalog designator) every night for 5 minutes and find $\lesssim 5 \%$ changes in the signal amplitude over the course of the project. The changes likely result from pointing offsets and inaccuracies in the opacity model. The accuracy of our absolute calibration will affect some of our parameter estimates (such as column density) in excess of our derived uncertainties $(\$ 3.5)$. The relative calibration within a spectrum appears to be better than the noise level in all the spectra of NH3SRC 47.

We subtract a linear baseline from each of the spectra, restricting to windows outside the expected range for ammonia emission from Perseus $\left(v_{L S R}=-2 \rightarrow 12 \mathrm{~km} \mathrm{~s}^{-1}\right)$, including the splitting from the hyperfine structure. The velocity window comes from the COMPLETE observations of ${ }^{13} \mathrm{CO}$ towards the Perseus cloud (Ridge et al. 2006).

The 193 targets were drawn, in order of precedence from (1) the locations of millimeter cores in the Bolocam survey of the region (Enoch et al. 2006) (2) the locations of submillimeter cores in the SCUBA survey of the region (Kirk et al.|2006), (3) sources in the literature survey of Jijina et al. (1999), and (4) cold, high-column-density objects in the dust map produced by Schnee et al. (in preparation), and (5) weak detections that appear in both the Bolocam and SCUBA maps but were not included in the published catalogs. Pairs of sources separated by less than than $31^{\prime \prime}$ (the GBT beam FWHM) were reexamined and a single source was selected for observation based on (sub)millimeter brightness. Table 1 summarizes the number of sources in each category and their detection fractions. Many of the same submillimeter cores are identified in both the SCUBA and the Bolocam surveys of the region. When analysis of the higher-resolution SCUBA map revealed sub-structure within a core identified as a single object in the Bolocam map, we omitted the Bolocam source and observed the substructure identified in the SCUBA catalog. The locations of the sources are shown in Figures 1 and 2, Nearly all of the (sub)millimeter sources are detected in ammonia emission with the exception of NH3SRCs 143 and 184 (BOLOCAM sources 90 and 120, Enoch et al. 2006). Both of these millimeter sources are marginal detections. We detect ammonia at several positions without significant millimeter emission, notably along 23 of the lines of sight selected based on their dust emission in the far infrared. Typical detections range from 0.5 to $4 \mathrm{~K}$ on the $T_{m b}$ scale. The noise levels in the spectra range from 40 to $150 \mathrm{mK}$, depending on integration time. The noise values are determined from the off-line regions of

${ }^{5}$ http://www.gb.nrao.edu/\$ sim\$rmaddale/Weather/index.html 
the spectra. Off-line regions are established iteratively as the regions more than 100 channels $\left(1.9 \mathrm{~km} \mathrm{~s}^{-1}\right)$ from significant $\left(3 \sigma_{r m s}\right)$ emission.

The $\mathrm{C}_{2}^{34} \mathrm{~S}$ line was not detected along any of the lines of sight. Given the ISM isotopic ratio ${ }^{32} \mathrm{~S} /{ }^{34} \mathrm{~S} \sim 22$ (Wilson \& Rood 1994), the lack of any detections, particularly when the $\mathrm{C}_{2} \mathrm{~S}$ line is strong suggests that the main line is usually optically thin. For NH3SRC 42, we establish a lower limit on the line ratio of 11.7 (the maximum in our population) by setting the $\mathrm{C}_{2}^{34} \mathrm{~S}$ amplitude to the $3 \sigma$ limit. Assuming excitation conditions are the same for both isotopomers, this implies $\tau_{\mathrm{CCS}}<1.4$ for one of the brightest $\mathrm{C}_{2} \mathrm{~S}$ lines in our sample.

In Figure 3 we show three spectra from our sample. Source 47 was observed every night as a consistency check on our flux calibration and is thus the best observed ammonia source in our sample (integrated $\mathrm{S} / \mathrm{N}$ of 530). Source 89 shows a typical narrow line spectrum $\left(\sigma_{v}=0.12 \mathrm{~km} \mathrm{~s}^{-1}\right)$ illustrating the resolution of the data. Source 31 is a multi-component spectrum which must be analyzed in more detail (see $\$ 3.4$ ). In Source 31, the multiple components are also visible in the $\mathrm{NH}_{3}(2,2)$ and $\mathrm{C}_{2} \mathrm{~S}$ lines.

We present a summary of our observations in Table 2. The properties of the cross-referenced names of the submillimeter cores are given in Enoch et al. (2006) and Kirk et al. (2006) respectively. Since ammonia has several hyperfine components that are well-separated in velocity, the integrated intensities reported for the $(1,1)$ and the $(2,2)$ lines are the sum of the integrated intensities over all channels that are within $3 \mathrm{~km} \mathrm{~s}^{-1}$ of any hyperfine component. We subtract the mean intensity in the off-line channels between the hyperfine components from the intensity of each channel in the on-line windows to offset any low-lying baseline residuals. When there is no ammonia emission or $\mathrm{C}_{2} \mathrm{~S}$ from which the line velocity can be determined, the main component of the ammonia line is assumed to lie at the the mean ${ }^{13} \mathrm{CO}$ velocity along the line of sight, as derived from the COMPLETE ${ }^{13} \mathrm{CO}$ data.

\section{PHYSICAL PARAMETER ESTIMATION}

Here we describe the estimation of physical parameters from the ammonia spectra. The method differs somewhat from previous work in that it forward models the properties of all observed spectral lines simultaneously given input physical properties. Then, the physical properties are derived using a non-linear least squares minimization code to determine the optimal fit to the observed spectrum. This stands in contrast with the standard method of obtaining these properties which relies on measuring line ratios and using these line ratios to calculate the physical properties. While the results should be the same using the two methods, the primary advantage of using a non-linear least squares fit is the automatic determination of uncertainties in the derived physical parameters as well as the covariances among those parameters (provided failures in the assumptions of the least-squares problem are appropriately accounted for). The model is optimized simultaneously for all observed spectra, which eliminates many systematic effects that arise from comparing properties derived from spectra separately. An additional advantage of this approach is that progressively more sophisticated models (e.g. De Vries \& Myers 2005) can be introduced to model specific spectral features. However, for this survey, we adopt a relatively simple model that can be applied to cores in a variety of environments.
For the spectra from a single object, a simple model is developed: the emission is assumed to arise from a homogeneous slab with uniform gas temperature, intrinsic velocity dispersion and uniform excitation conditions for all hyperfine transitions of the $\mathrm{NH}_{3}$ lines. Detailed studies of $\mathrm{NH}_{3}$ emission in conjunction with other molecular tracers illustrate the shortcomings of this model. The observations of Ladd et al. (1994) suggest that the ammonia emission is not completely uniform on $30^{\prime \prime}$ scales. Tafalla et al. (2004) note that the kinetic temperature of the two cores they study in detail is constant while there are radial variations in ammonia abundance and excitation temperatures in cores. Mauersberger et al. (1988) find the line width of the $\mathrm{NH}_{3}(2,2)$ transitions to be larger than the $\mathrm{NH}_{3}(1,1)$ transitions in the high-mass star forming region $\mathrm{W} 3$.

Despite these limitations, the uniform slab model is still useful. Lacking information about the spatial distribution of ammonia, a uniform slab is the simplest model we can adapt which should provide reasonable average properties over the region within the beam (see, for example, the conclusions of Tafalla et al. 2004). The GBT beam is roughly half the typical core radius for objects in Perseus Jijina et al. (1999, \$2), so the emission should couple well to the GBT beam (hence our adoption of the main beam temperature scale). Separate fits to the line width of the strong $\mathrm{NH}_{3}(1,1)$ and $(2,2)$ detections show that the $(2,2)$ line is, on average $(8 \pm 2) \%$ wider than the $(1,1)$ line. Several spectra show evidence for non-uniform excitation of ammonia hyperfine components. However, we emphasize that these deviations in excitation and line width are small (a few percent) and are only apparent because of the high quality of the data. In general, the slab model produces high-quality fits to the data. More complicated spectra (see Figure 4 merit further investigation and these interesting sources will be investigated in more detail elsewhere. This presentation of the data is restricted to generating average properties based on the simple model for comparison of physical properties across the sample.

\subsection{Standard Spectral Model}

Our GBT reduction pipeline produces three spectra with $1.846 \mathrm{kHz}$ resolution centered on the $\mathrm{NH}_{3}(1,1), \mathrm{NH}_{3}(2,2)$ and $\mathrm{C}_{2} \mathrm{~S}\left(2_{1} \rightarrow 1_{0}\right)$ transitions. The spectra are on the $T_{A}^{*}$ scale which include corrections for atmospheric opacity. We derive the physical parameters for a simple ammonia system: the gas is assumed to have a slab geometry with uniform properties, in particular gas kinetic temperature. The model assumes that column density of the material has a Gaussian distribution in velocity with a dispersion of $\sigma_{v}$ around an LSR velocity centroid $v_{L S R}$ :

$$
\frac{d N}{d v} d v=N_{0} \exp \left[-\frac{\left(v-v_{L S R}\right)^{2}}{2 \sigma_{v}^{2}}\right] .
$$

The ammonia $(1,1)$ and $(2,2)$ lines have 18 and 21 hyperfine components respectively so the optical depth implied by the column density distribution is split among each of the hyperfine components. As such, the opacity distribution for the $(1,1)$ and $(2,2)$ lines can be written (in terms of frequency on the sky with respect to the LSR) as:

$$
\tau(\nu)=\tau_{1} \sum_{i=1}^{18} s_{i} \exp \left[-\frac{\left(\nu-\nu_{i}-\nu_{L S R}\right)^{2}}{2 \sigma_{i}^{2}}\right]
$$


TABLE 1

SUMMARY OF SOURCE ORIGINS

\begin{tabular}{cccccc}
\hline \hline Origin & Abbrev. & Number & $\mathrm{NH}_{3}(1,1)$ Det. Frac. & $\mathrm{NH}_{3}(2,2)$ Det. Frac. & $\mathrm{C}_{2}$ S Det. Frac. \\
\hline Bolocam & $\mathrm{B}$ & 115 & $98 \%$ & $85 \%$ & $67 \%$ \\
SCUBA & $\mathrm{S}$ & 16 & $100 \%$ & $94 \%$ & $56 \%$ \\
Literature & $\mathrm{L}$ & 5 & $100 \%$ & $80 \%$ & $100 \%$ \\
Dust & $\mathrm{D}$ & 38 & $61 \%$ & $8 \%$ & $13 \%$ \\
Weak Submm & $\mathrm{W}$ & 19 & $26 \%$ & $11 \%$ & $16 \%$ \\
\hline Total & $\cdots$ & 193 & $84 \%$ & $63 \%$ & $51 \%$ \\
\hline
\end{tabular}

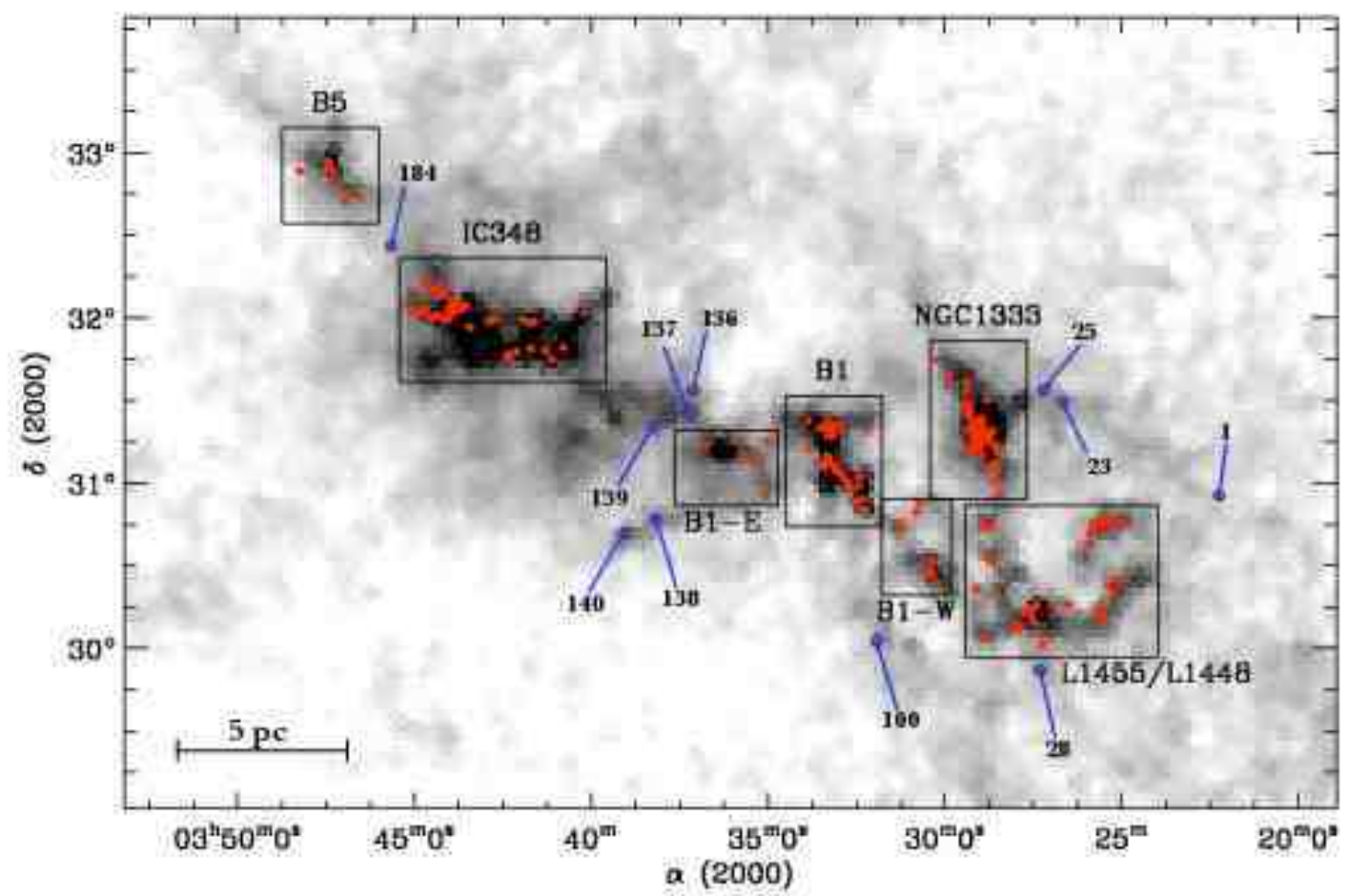

FIG. 1. - Locations of GBT pointings (red points) overlaid upon a map of extinction for the Perseus region. Seven subregions have been defined and have their sources labeled in Figure 2 For sources outside the defined subregions, the sources are labeled on the map with the number of the source given in Table 2 The extinction map is derived from applying the NICER algorithm to 2MASS data (Ridge et al. 2006) and the grayscale has a square-root transfer function running from $A_{V}=0$ to $7 \mathrm{mag}$.

$$
+\tau_{2} \sum_{j=1}^{21} s_{j} \exp \left[-\frac{\left(\nu-\nu_{j}-\nu_{L S R}\right)^{2}}{2 \sigma_{j}^{2}}\right] .
$$

where $\nu_{L S R}$ is determined by the Doppler formula (radio convention):

$$
\nu_{L S R}=\left(1-\frac{v_{L S R}}{c}\right) \nu_{\text {rest }} .
$$

Above, $\tau_{1}\left(\tau_{2}\right)$ is the total opacity in the $(1,1)[(2,2)]$ line transition; and $s_{i}\left(s_{j}\right)$ is statistical weight of the $i$ th $(j$ th) hyperfine component of the $(1,1)[(2,2)]$ transition. Each component has a sky frequency $\nu_{i}\left(\nu_{j}\right)$ and a corresponding width $\sigma_{i}\left(\sigma_{j}\right)$ given by

$$
\sigma_{i}=\frac{\sigma_{v}}{c} \nu_{i}
$$

The number of molecules found in states that undergo the $(1,1)$ vs. the $(2,2)$ inversion transitions is governed by the rotation temperature of the system $\left(T_{R}\right)$. Specifically, the population ratio is established by the magnitude of $T_{R}$ relative to the energy gap between the two states which (expressed in K) is

$T_{0}=41.5 \mathrm{~K}$. The population ratio is given by the Boltzmann factor and the statistical weights of the two states $[g(1,1)=3$ and $g(2,2)=5]$. We assume that the transitions have equal line widths, $\sigma_{v}(1,1)=\sigma_{v}(2,2)$, and excitation temperatures, $T_{x}(1,1)=T_{x}(2,2)$. After including the amplitudes of the dipole matrix elements (e.g. $|\mu(1,1)|^{2}$ ), the ratio of the opacities can be expressed as (Ho et al. 1979; Ho \& Townes 1983):

$$
\begin{aligned}
\frac{\tau_{2}}{\tau_{1}} & =\left[\frac{\nu_{(2,2)}}{\nu_{(1,1)}}\right]^{2} \frac{\sigma_{v}(1,1)}{\sigma_{v}(2,2)} \frac{T_{x}(1,1)}{T_{x}(2,2)} \frac{|\mu(2,2)|^{2}}{|\mu(1,1)|^{2}} \frac{g(2,2)}{g(1,1)} \exp \left(-\frac{T_{0}}{T_{R}} \oint_{5}\right) \\
& =\left[\frac{\nu_{(2,2)}}{\nu_{(1,1)}}\right]^{2} \frac{20}{9} \exp \left(-T_{0} / T_{R}\right) .
\end{aligned}
$$

We assume that the kinetic temperature $\left(T_{k}\right)$ is much less than $T_{0}$, implying that the $(1,1)$ and $(2,2)$ states are the only populated rotational levels of the ammonia system. Thus, it is a two-state system for which the kinetic temperature can be related to the rotation temperature with knowledge of the collision coefficients using detailed balance arguments 

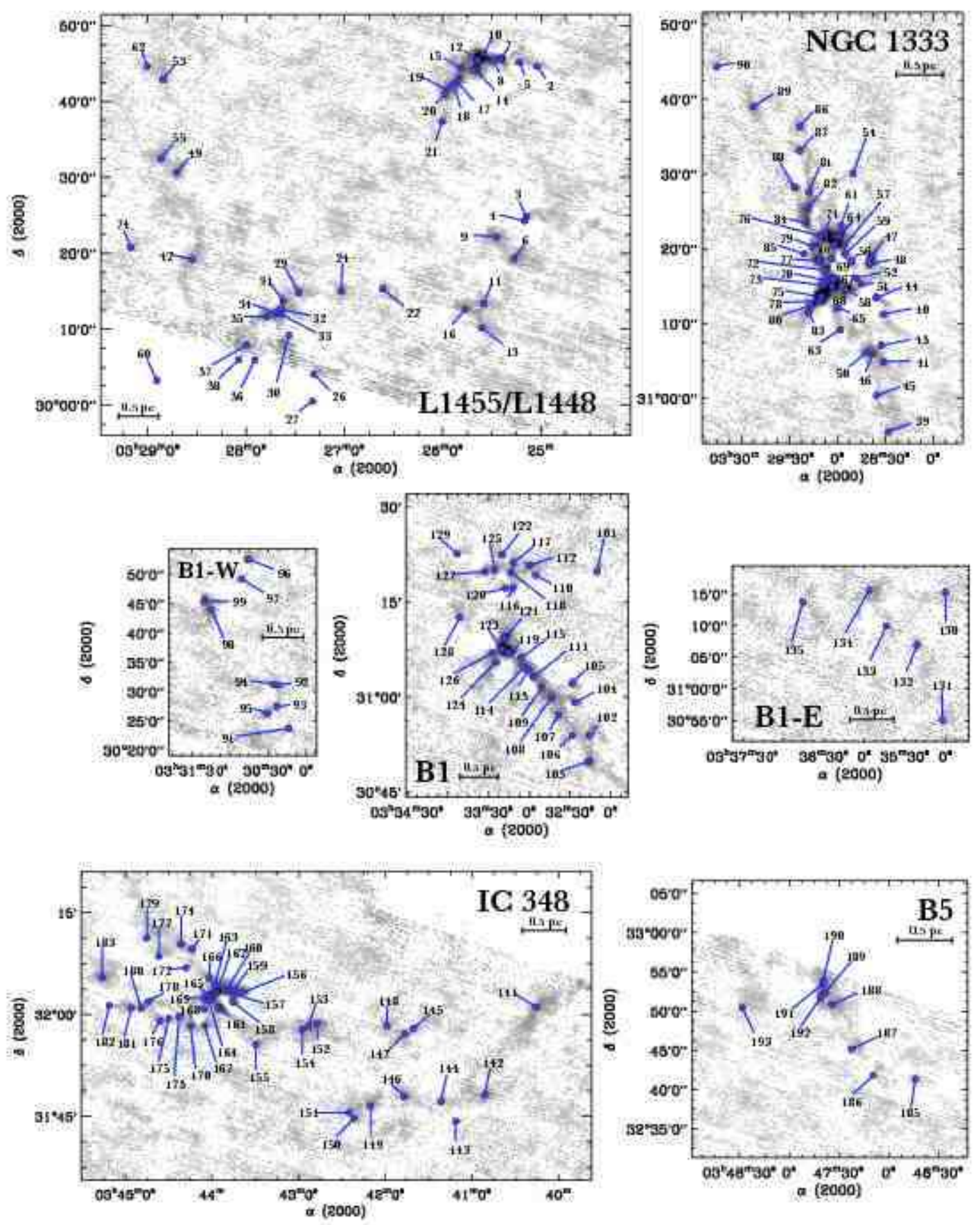

FIG. 2.- Locations of GBT pointings (red points) overlaid upon a maps of millimeter emission from the BOLOCAM survey of the region (Enoch et al.2006). The seven maps correspond to the subregions defined in Figure 1 Individual pointings are labeled with the number of the source given in Table 2 The grayscale has a square-root transfer function spanning $f_{\nu}=0$ to $400 \mathrm{mJy}^{\text {beam }}{ }^{-1}$.

(Swift et al. 2005). In this case:

$$
T_{R}=T_{k}\left\{1+\frac{T_{k}}{T_{0}} \ln \left[1+0.6 \exp \left(-15.7 / T_{k}\right)\right]\right\}^{-1} .
$$

Finally, given a radiation excitation temperature, $T_{x}$, the two ammonia spectra can now be modeled in their entirety:

$$
T_{A}^{*}(\nu)=\eta_{m b}(\nu) \eta_{f}\left[J\left(T_{x}\right)-J\left(T_{b g}\right)\right]\left[1-e^{-\tau(\nu)}\right]
$$

where $\eta_{m b}(\nu)$ is the main beam efficiency of the GBT $(\approx 0.81$ for the frequency range in this study), $\eta_{f}$ is the filling fraction of the emission in the beam, $T_{b g}=2.73 \mathrm{~K}$ and

$$
J(T)=\frac{h \nu}{k} \frac{1}{\exp (h \nu / k T)-1} .
$$

After making assumptions about source-beam coupling and filling fraction, the spectrum is entirely determined by five parameters: $T_{k}, T_{x}, \tau_{1}, \sigma_{v}$, and $v_{L S R}$. Alternatively, we can assume 


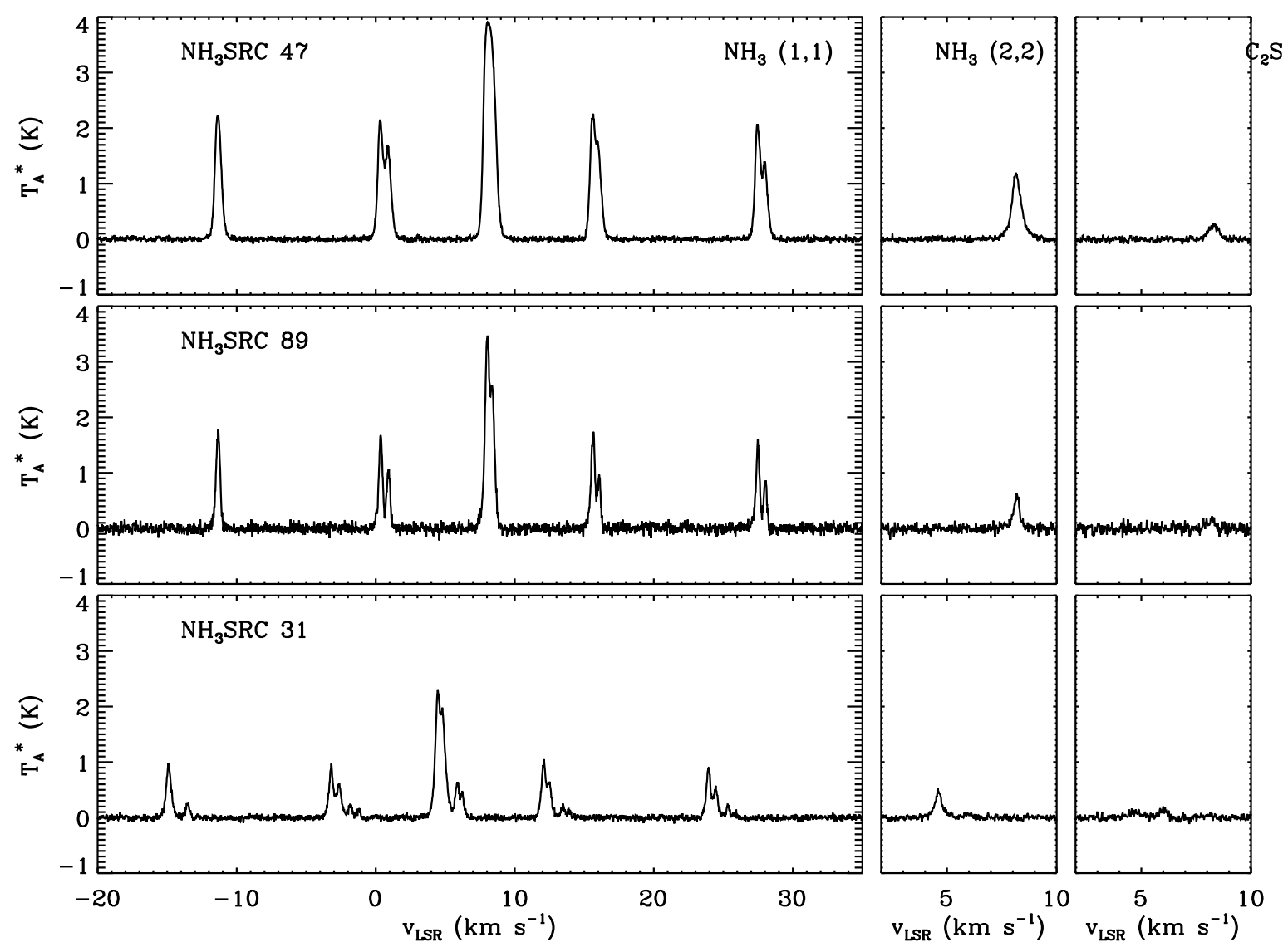

FIG. 3.- Three sample spectra from the ammonia sample including the source used for a flux check (Source 47), a narrow-line spectrum (Source 89) and a multicomponent spectrum (Source 31).

that $T_{x}=T_{k}$ (LTE) and let the filling fraction $\eta_{f}$ vary.

In addition to the ammonia system, we also measure spectra for the $\mathrm{C}_{2} \mathrm{~S}$ line. We also fit a three parameter Gaussian to the $\mathrm{C}_{2} \mathrm{~S}$ line simultaneously while deriving parameters for the ammonia spectra. The total spectral model is given by:

$T_{A}^{*}(\operatorname{model})=T_{A}^{*}\left(\mathrm{NH}_{3}\right)+\eta_{m b}(\nu) T_{\mathrm{CCS}} \exp \left[-\frac{\left(\nu-\nu_{0}-\nu_{L S R}-\nu_{o f f}\right)^{2}}{2 \sigma_{\mathrm{CCS}}^{2}}\right]$

Here, $\nu_{0}$ is the given rest frequency of the $\mathrm{C}_{2} \mathrm{~S}$ line, $\nu_{L S R}$ is the appropriate frequency shift account for motion with respect to the LSR using the same LSR velocity as derived from $\mathrm{NH}_{3}$. We define a velocity offset $V_{\text {off }}$ which, via the Doppler formula, relates to the derived frequency offset $\nu_{o f f}$. $T_{\mathrm{CCS}}$ and $\sigma_{\mathrm{CCS}}$ are the amplitude and derived width of the line. We do not know the excitation temperature of the $\mathrm{C}_{2} \mathrm{~S}$ line, but we assume the excitation is similar to that of ammonia since the critical density $\left(n_{c r} \sim 10^{4.5} \mathrm{~cm}^{-3}\right.$, Langer et al. 1995) is close to that of the ammonia lines (Swade 1989). The $\mathrm{C}_{2} \mathrm{~S}$ line complex adds the parameters $T_{\mathrm{CCS}}, \sigma_{\mathrm{CCS}}$ and $V_{\text {off }}$ to our fit.

The final effect we account for is the sampling of the GBT correlation spectrometer. The lags in the spectrometer are weighted uniformly (i.e. no online smoothing). As a result, a channel has a nominal profile of a sinc function:

$$
\phi(\nu)=\frac{\sin \left[\pi\left(\nu-\nu_{k}\right) / \Delta \nu\right]}{\pi\left(\nu-\nu_{k}\right) / \Delta \nu} .
$$

Here, $\nu_{k}$ is the channel center and $\Delta \nu$ is the channel spacing. After deriving $T_{A}^{*}$ (model), we digitally sample it at one-fourth the channel spacing and we convolve the model spectrum with channel profile to produce the final result which is compared with observations.

We find the maximum likelihood model for the spectrum using a non-linear least-squares fitting routine ${ }^{6}$. The optimization occurs in two steps: first the fit is performed to the entirety of the $\mathrm{NH}_{3}(1,1), \mathrm{NH}_{3}(2,2)$ and $\mathrm{C}_{2} \mathrm{~S}$ spectra. If emission is detected the fit is performed again, ignoring regions of the spectrum more than $2.5 \mathrm{~km} \mathrm{~s}^{-1}$ away from significant emission using the results of the first fit as an initial guess for the optimization. This second step reduces the number of noise-only channels in the fit and allows for a cleaner convergence to an optimal set of properties. Examples of the fitting appear in Figure 4. We have chosen four representative spectra for several cases found in the single-component models. The first two columns of the figure show the successful applications of the model in the regular and low optical depth regimes $(\$ 3.2)$. The final two columns of the figure show the slight deviations frequently encountered in spectra with high optical depth in the lines. The slight deviations may be the result of non-LTE excitation of the different hyperfine components. Several other spectra show asymmetries in the line profiles for which a Gaussian model of the velocity distribution is inaccurate (see $\$ 3.4$ for further discussion of these cases). The full set of spectra for lines of sight with detections are available as online-only figures (Figure 6a-6ff) and are downloadable from the COMPLETE website ${ }^{7}$.

${ }^{6}$ C. Markwardt's MPFIT package. Note that, since the channels are not independent, strict least squares fitting is technically inappropriate. See $\$ 3.5$

7 http://www.cfa.harvard.edu/COMPLETE/data_html_pages/GBT_NH3 


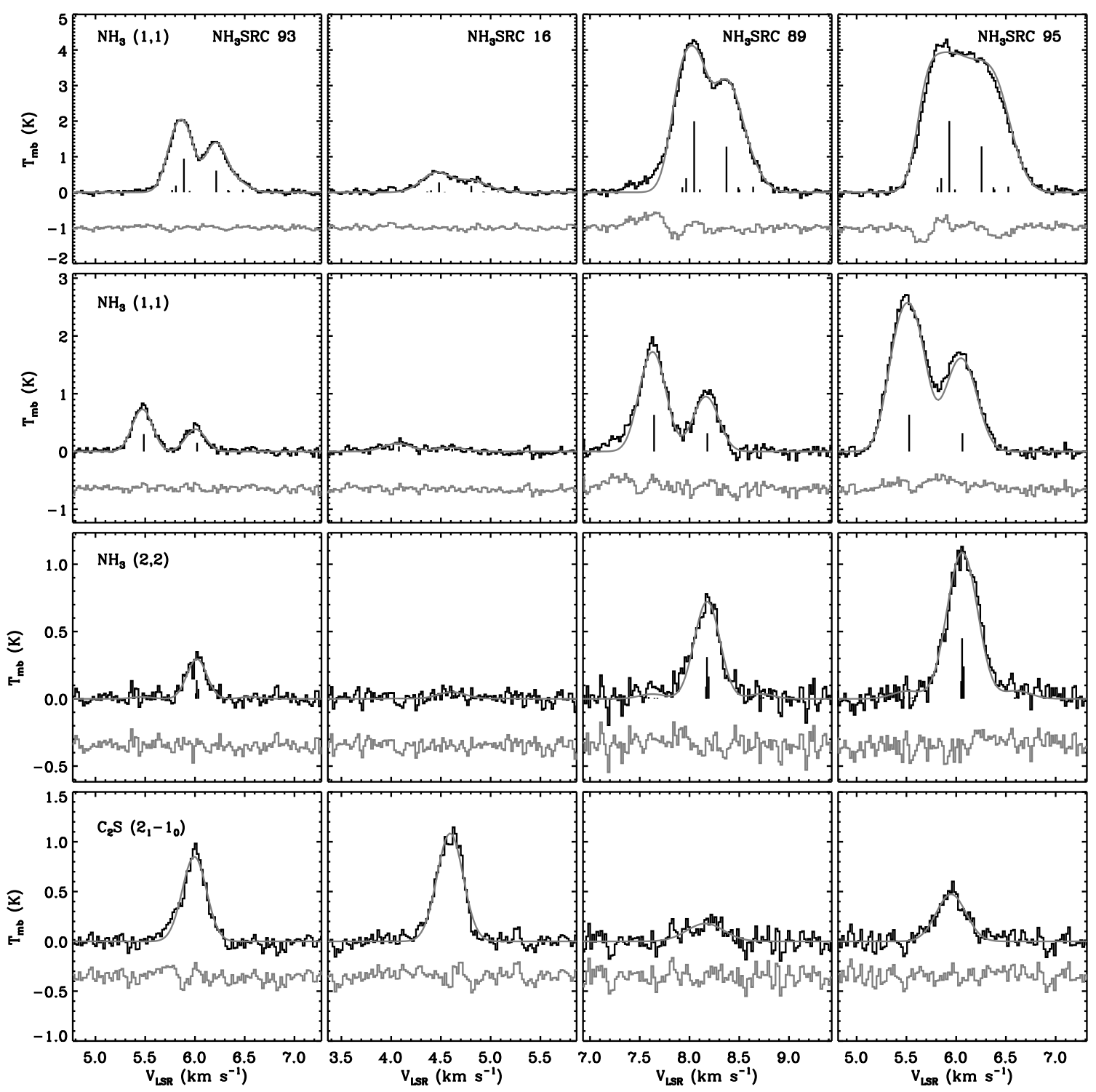

FIG. 4.- Examples of fits to four GBT spectra. Each row shows a different excerpt from the spectra including (in order) the main component of the ammonia $(1,1)$ complex, the satellite complex at $+19.85 \mathrm{~km} \mathrm{~s}^{-1}$ relative to the main complex (the $F_{1}, F=0,1 / 2 \rightarrow 1,1 / 2$ transition; the $F_{1}, F=0,1 / 2 \rightarrow 1,3 / 2$ transition is also visible, offset by $-0.54 \mathrm{~km} \mathrm{~s}^{-1}$ ), the main $(2,2)$ complex and the $\mathrm{C}_{2} \mathrm{~S}$ line. The gray, smooth line shows the model spectrum and the residual is shown below each profile. Each column shows a different object including source 93, chosen for a good fit, source 16 chosen for a low optical depth fit, and sources 89 and 95 which show the slight deviations from the amplitude of the model in the case of high optical depth. The velocities and relative strengths of the $\mathrm{NH}_{3}$ hyperfine structure are indicated with vertical lines.

\subsection{Low Optical Depth Regime}

When $\tau(\nu) \ll 1$ for the ammonia complex over all $\nu$, the parameters $T_{x}$ and $\tau_{11}$ become degenerate and it is impossible to solve for the two parameters independently. In this case, we expand Equation 8 assuming the Rayleigh-Jeans limit so that

$$
T_{A}^{*}(\nu)=\eta_{m b}(\nu) \eta_{f}\left(T_{x}-T_{b g}\right) \tau(\nu)
$$

where $\tau(\nu)$ is given by Equation 2 In this case, we optimize the fit for the free parameter $\gamma \equiv\left(T_{x}-T_{b g}\right) \tau_{1}$ and the ammonia spectrum is determined by four free parameters: $T_{k}, \gamma, \sigma_{v}$, and $v_{L S R}$. This approximation is accurate to better than $10 \%$ for all components of the ammonia complex provided $\tau_{1}<1$.
We measure the total column density of $\mathrm{NH}_{3}$ and $\mathrm{C}_{2} \mathrm{~S}$ using the derived parameters from the fit. For example, the column density in the $\mathrm{NH}_{3}(1,1)$ state is (e.g. Rohlfs \& Wilson 2004):

$$
N(1,1)=\frac{8 \pi \nu_{0}^{2}}{c^{2}} \frac{g_{1}}{g_{2}} \frac{1}{A_{(1,1)}}\left[1-\exp \left(\frac{h \nu_{0}}{k T_{x}}\right)\right]^{-1} \int \tau(\nu) d \nu(13)
$$

The statistical weights of the upper and lower levels of the inversion transition are equal. The Einstein A value for the inversion transition is $A_{(1,1)}=1.68 \times 10^{-7} \mathrm{~s}^{-1}$ (Pickett et al. 1998). The opacity per unit frequency is given in terms of the total opacity of the line $\left(\tau_{1}\right)$ by the first term in Equation 2.

$$
\text { 3.3. Column Density Estimates } \quad \int \tau(\nu) d \nu=\tau_{1} \int d \nu \sum_{i} s_{i} \exp \left[-\frac{\left(\nu-\nu_{i}-\nu_{L S R}\right)^{2}}{2 \sigma_{i}^{2}}\right]
$$




$$
=\sqrt{2 \pi} \sigma_{i} \tau_{1}=\sqrt{2 \pi} \frac{\sigma_{v}}{c} \nu_{0} \tau_{1}
$$

under the approximation that the frequencies of the individual hyperfine components are the same $\left(\nu_{0}\right)$. The partition function for the metastable states $(J=K)$ of ammonia is given by (Rohlfs \& Wilson 2004):

$$
Z=\sum_{J}(2 J+1) S(J) \exp \left\{\frac{-h\left[B J(J+1)-(C-B) J^{2}\right]}{k T_{K}}\right\}
$$

Here, $B$ and $C$ are the rotational constants of the ammonia molecule: $298117 \mathrm{MHz}$ and $186726 \mathrm{MHz}$ respectively (Pickett et al. 1998). The factor $S(J)$ equals 2 for $J=3,6,9 \ldots$ and 1 otherwise, accounting for the extra statistical weight of ortho- $\mathrm{NH}_{3}$ over para- $\mathrm{NH}_{3}$. Owing to the relatively short lifetime of the $J \neq K$ states, we assume all ammonia molecules are in the metastable states. To determine the total column density, we scale the column density in the $(1,1)$ state by $Z / Z(J=1)$. We truncate the partition function at 50 terms.

For the low optical depth case, we expand the exponential in Equation 13 .

$$
\begin{aligned}
N(1,1) & =\frac{8 \pi k \nu_{0}}{h c^{2}} \frac{1}{A_{(1,1)}} \int T_{x} \tau(\nu) d \nu \\
& =\frac{8 \pi k \nu_{0}^{2}}{h c^{3}} \frac{1}{A_{(1,1)}} \sqrt{2 \pi} \sigma_{v}\left(T_{x}-T_{b g}\right) \tau_{1}
\end{aligned}
$$

where $\sigma_{v}$ and $\left(T_{x}-T_{b g}\right) \tau_{1}$ are determined by the low-opticaldepth fit (\$3.2).

We also estimate the column density of $\mathrm{C}_{2} \mathrm{~S}$ assuming that the line is optically thin, an assumption bolstered by our failure to detect $\mathrm{C}_{2}{ }^{34} \mathrm{~S}$ along any of the lines-of-sight.

$$
N\left(\mathrm{C}_{2} \mathrm{~S}, J=1\right)=\frac{8 \pi k \nu_{0}}{h c^{2}} \frac{g_{1}}{g_{2}} \frac{1}{A_{21}} \sqrt{2 \pi} \sigma_{v}\left(T_{x}-T_{b g}\right) \tau_{21} .
$$

Here, $g_{J}=2 J+1, A_{21}=5.44 \times 10^{-7} \mathrm{~s}^{-1}$ (Pickett et al. 1998) and we take $\left(T_{x}-T_{b g}\right) \tau_{21}=T_{\mathrm{CCS}}$. Again, we calculate the total column density of $\mathrm{C}_{2} \mathrm{~S}$ using the partition function. For a state with energy above the ground state $E_{i}$ and degeneracy $g_{i}$, the partition function is the standard

$$
Z=\sum_{i} g_{i} \exp \left[-\frac{E_{i}}{k T_{k}}\right] \text {. }
$$

We adopt the values of $E_{i}$ and $g_{i}$ from the tabulated molecular data in the JPL molecular spectroscopy catalog (Pickett et al. 1998) and assume the 295 states are thermally populated. It may not be appropriate to use the kinetic temperature derived from the $\mathrm{NH}_{3}$ for the $\mathrm{C}_{2} \mathrm{~S}$ partition function since the two species may not be thermally coupled. This systematic effect limits our ability to measure the $\mathrm{C}_{2} \mathrm{~S}$ column. For both molecules, the uncertainties in the column density are established by adding a normal deviate times the uncertainty to the input parameters and recalculating the column densities. After repeating this redistribution within the errors a large number of times, the error are determined from the width of the resulting distribution.

We tested the results of the uniform slab modeling by comparing the results to the values derived from the hyperfine fitting routines in the CLASS package. We checked the line width, opacity, excitation temperature and LSR velocity. The results were identical within the errors of our analysis except for complex source spectra (e.g. asymmetric profiles, multiple components).

\subsection{Multi-Component Fitting}

Several of the spectra show significant velocity structure in the line beyond what is expected from the hyperfine structure of ammonia (see, for example, Source 31 in Figure 3). In cases where the number of components is readily modeled, we have attempted to fit a multicomponent model to the spectrum. In this model, we assume that there are two objects in the beam each with $\eta_{f}<1$ and we operate in the LTE approximation $\left(T_{x}=T_{k}\right)$. Hence, it is not necessary to calculate radiative transfer effects of one component through another. The approximation appears to be sufficiently good for our purposes.

We only present multiple components to the data where the evidence is unambiguous that a multiple-component fit is appropriate. This means two clear peaks in the $\mathrm{NH}_{3}(2,2)$ line. In some cases the velocity separation is sufficient that multiple components are also well distinguished in the $(1,1)$ line. The initial conditions for the fit are established by hand, but the optimization is performed simultaneously for both components. To prevent run-away solutions, we constrain the initial velocities to be within $0.1 \mathrm{~km} \mathrm{~s}^{-1}$ of the initial guess and also constrain the $\mathrm{C}_{2} \mathrm{~S}$ and $\mathrm{NH}_{3}$ velocities to be within $0.1 \mathrm{~km} \mathrm{~s}^{-1}$ for each of the components. We report the fits to the multiple components independently in Table 3 appending a decimal and the number of the component onto source name. In the six spectra with multiple components reported, all show that the sum of the their filling fractions is less than unity. The typical filling fraction for a single component is $\eta_{f}=0.3$.

Seven additional spectra show strong evidence for multiple components in some or all of the lines. In particular, there are often multiple components in the $\mathrm{C}_{2} \mathrm{~S}$ and a broad or poorly fit $\mathrm{NH}_{3}(1,1)$ line but no clear evidence for multiple components in the $\mathrm{NH}_{3}(2,2)$ line. Since we cannot be certain that these two components in $\mathrm{C}_{2} \mathrm{~S}$ are physically associated with two components in $\mathrm{NH}_{3}$, we refrain from performing a multicomponent fit but note the presence of the components in Table 3 . There are a number of additional spectra with weaker evidence of multiple components, or evidence for more than two components. We likewise flag these objects in Table 3

\subsection{Uncertainties and Limits in Derived Parameters}

The reported uncertainties in Table 3 are the derived uncertainties from the nonlinear least-squares fitting using the covariance matrix. The individual channels are not independent (the channel width is $1.525 \mathrm{kHz}$ and the resolution is 1.862 $\mathrm{kHz}$ ) which violates an assumption of least-squares fitting. The errors determined reported from the covariance matrix represent the $\Delta \chi^{2}=1$ ellipsoid. To determine appropriate uncertainties, we generated multiple realizations of several different spectral models each with different noise distributions. We used our fitting routine to derive parameters in the presence of noise and compared the distribution of the derived parameters to the input model parameters. Over all the different spectral models, the true errors were a factor of $\leq 1.6$ larger than the errors derived from the covariance matrix assuming the data were independent. While not technically a least-squares fit, accurate confidence intervals for the derived parameters can still be estimated using the $\Delta \chi^{2}=2.56=1.6^{2}$ surface (Press et al. 1992). We have investigated the shape of $\chi^{2}$ space in our data and find that the region around the minimum $\chi^{2}$ is well approximated by a paraboloid. To report errors consistent with a $1 \sigma$ spread around the derived parameters, we scale the reported errors up by a factor of 1.6. 
The covariance matrix also indicates which parameters are correlated with each other in the fits. The additional uncertainty due to this correlation is accounted for in the reported errors. For parameters $i$ and $j$, we express the covariance in terms of the normalized covariance: $\sigma_{i j} /\left(\sigma_{i} \sigma_{j}\right)^{1 / 2}$. We examined the average covariance matrix over the 133 fits with sufficiently strong $\mathrm{NH}_{3}(1,1)$ emission to fit for optical depth and excitation temperature separately. We find that the most obvious (anti)correlation is between $T_{e x}$ and $\tau: \sigma_{i j} /\left(\sigma_{i} \sigma_{j}\right)^{1 / 2}=$ -0.95 . This strong anticorrelation necessitates the low opacity treatment described in $\$ 3.2$. The excitation temperature is also correlated with $T_{K}(0.18)$ and $\sigma_{v}(0.24)$. The line opacity $\left(\tau_{i}\right)$ has an anticorrelation with the line width $\sigma_{v}(-0.49)$ and $T_{k}(-0.23)$. For the $\mathrm{C}_{2} \mathrm{~S}$ line, there is the anticorrelation between the amplitude $\left(T_{\mathrm{CCS}}\right)$ and the line width $\sigma_{\mathrm{CCS}}$ typical of Gaussian fits $(-0.57$ in our case). The other elements of the covariance matrix are consistent with zero.

The uncertainties do not reflect the overall uncertainty in the amplitude scale calibration which is $\sim 5 \%$, The relative calibration across the spectra is much better than $5 \%$, so certain properties are unaffected by the overall amplitude calibration. The integrated intensities reported in Table 2 as well as properties derived from the amplitude of the emission (column densities, excitation temperatures, filling fractions, and antenna temperatures) have $\sim 5 \%$ uncertainties. In contrast, line-of-sight velocities, line widths, optical depths and kinetic temperatures (since the latter two are driven by line ratios) have uncertainties close to their reported precisions.

In several cases, we detect the $(1,1)$ transition of $\mathrm{NH}_{3}$ but not the $(2,2)$ transition. When we can establish an upper limit on the intensity of $(2,2)$ line, we report a $3 \sigma$ upper limit on the temperature of the ammonia. Since the ammonia temperature is used in the calculation of column densities, the upper limit on temperature produces a lower limit on the $\mathrm{NH}_{3}$ column density since the partition function correction is a decreasing function of temperature for $T_{k}<40 \mathrm{~K}$. In contrast, the correction for $\mathrm{C}_{2} \mathrm{~S}$ is an increasing function of temperature so the upper limit of temperature creates an upper limit for the $\mathrm{C}_{2} \mathrm{~S}$ column density.

The velocity width of the ammonia complex is quoted as an upper limit in Table 3 for instances when (1) there are multiple components along the line of sight that cannot be decoupled and fit separately or (2) when the line widths are large but the signal-to-noise is small such that the broadening of the line cannot be distinguished from the splitting due to the hyperfine structure. In the latter case, the upper limit reported may represent the actual value, but we cannot distinguish between a large intrinsic line width and line widths that result from the hyperfine structure. We note that we find no cores with large ammonia $(1,1)$ antenna temperatures $\left(T_{A}^{*}>1 \mathrm{~K}\right)$, large line widths $\left(\sigma_{v}>0.2 \mathrm{~km} \mathrm{~s}^{-1}\right)$ which have no evidence for multiple components along the line of sight. Said differently, all large line width cores may have large line widths only because of multiple components along the line of sight.

The largest systematic in the reported values is the bias introduced by the uniform slab model presented above. Again, we emphasize that the model is adopted for uniform application to a large sample; individual spectra can be investigated in more detail. One difficulty in applying our model may occur in comparing the $(1,1)$ and $(2,2)$ emission. Although the critical densities for the two transitions are similar, the $\mathrm{NH}_{3}(1,1)$ emission has larger optical depths than the $(2,2)$ emission. In cores with radial gradients in tempera- ture, opacity effects may result in the $(2,2)$ emission revealing warmer gas than the $(1,1)$ emission. We have attempted fitting the most optically thick spectra with the hill models of De Vries \& Myers (2005), but we do not find a significant improvement in the fit quality with the additional complications the model entails. Such line-of-sight variations in the excitation conditions are ignored in our simple treatment, but our derived values should yield (appropriately weighted) average conditions along the line of sight.

\subsection{Comparison to Previous Work}

Ammonia has been observed towards Perseus in several previous studies. We compare the derived properties from our analysis to those values found in the literature for sources other studies have observed. For comparison, we use homogenized properties in the catalog of Jijina et al. (1999) which are drawn primarily from the work of Ladd et al. (1994); Bachiller \& Cernicharo (1986); Bachiller et al. (1987) and Juan et al. (1993). We find good agreement between our line widths and temperatures with $\lesssim 20 \%$ variations across most of our sources. Discrepant points are invariably found in NGC 1333 where larger line widths are typically found in earlier studies. We suspect that the larger beam sizes of previous work blend together more disparate emission than the GBT observations resulting in the larger line widths. The agreement with ammonia column density is less well established with variations up to $0.5 \mathrm{dex}$ are found. However, the largest discrepancies are associated with highly uncertain column densities flagged in Jijina et al. (1999). We conclude that these new data match the results of previous studies quite well with significant variations attributable to the improved quality of the observations.

\section{DISTRIBUTIONS OF DERIVED PROPERTIES}

In this section, we present a brief summary of the observed spectra and their derived properties. A graphical summary of the data that appear in Tables 2 and 3 is given in Figure 5. The first two panels show the typical distribution of line temperatures on the $T_{m b}$ scale for the (sub)millimeter (gray) and all other sources. The strongest sources are all associated with millimeter-bright objects and other targets are typically weak in $(1,1)$ emission and infrequently detected in $(2,2)$. Two sources, NH3SRC 27 and 60, are outside the bounds of either (sub)millimeter study but we detect significant line emission. We have included these in the millimeter-faint population since they are only associated with MIPS-derived dust features, but this assignment may be incorrect.

The derived intrinsic line widths are typically $<0.2 \mathrm{~km} \mathrm{~s}^{-1}$ across the entire sample with a high line width tail to the distribution. As noted previously, these line widths may be upper limits since in all cases where there is sufficient signalto-noise to resolve the structure of the line there is evidence for multiple velocity components. The typical (total) optical depth of the ammonia complex is $\sim 4$ and the main complex has a thickness half the optical depth shown. Hence, in most cases, the lines are only moderately opaque, though some line complexes are quite optically thick. The (sub)millimeterweak sources have a higher median line width and lower opacity than the (sub)millimeter-bright population.

The derived kinetic temperatures of the cores are uniformly cool $\left(\mathrm{T}_{K}<20 \mathrm{~K}\right)$ and are typically $11 \mathrm{~K}$, substantially lower than is assumed in some work (e.g. Kirk et al. 2006) for submillimeter cores. If the dust and gas are well-coupled, assuming $T=15 \mathrm{~K}$ for the Perseus cores can result in underes- 

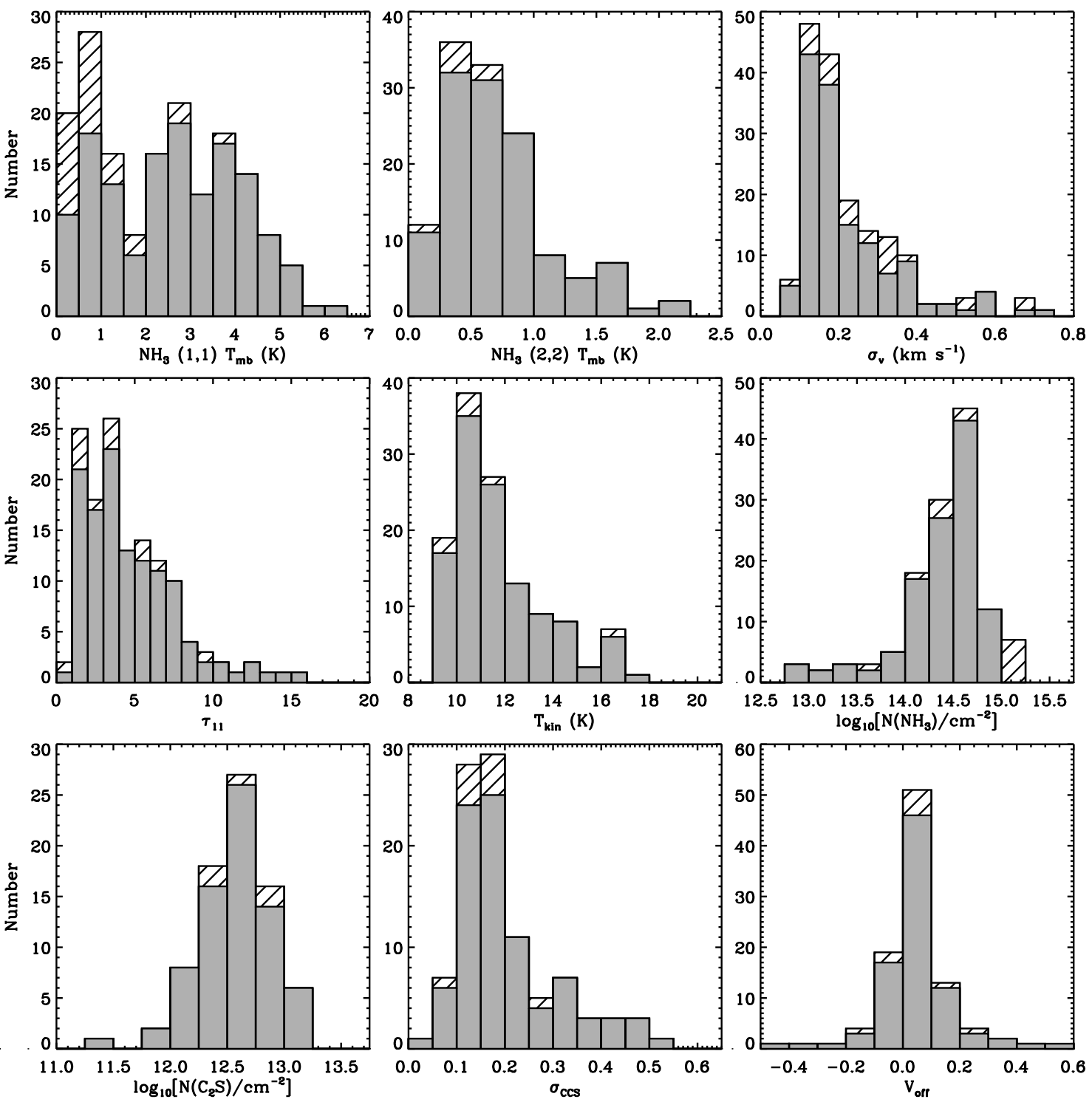

FIG. 5.- Distribution of the observed and derived properties for the GBT spectra. Working from left to right, top to bottom, the nine panels show the peak main beam temperatures for the $(1,1)$ and $(2,2)$ lines, the intrinsic velocity dispersion of $\mathrm{NH}_{3}$ derived from the (1,1) and (2,2) lines (including upper limits as data), the total optical depth of the $(1,1)$ complex, the kinetic temperature of the gas, the column density of the ammonia emission, the column density of the $\mathrm{C}_{2} \mathrm{~S}$, the velocity dispersion of the $\mathrm{C}_{2} \mathrm{~S}$ line, and the velocity offset of the $\mathrm{C}_{2} \mathrm{~S}$ line from the ammonia complex. The gray histogram shows the distributions of properties for the pointings associated with (sub)millimeter sources and the hashed histogram shows all remaining objects. Objects are included in the histograms only if they have well-determined values for the properties reported (with the exception of line width).

timating the mass of the cores by a factor of 1.7 . However, assuming a temperature of $10 \mathrm{~K}$ yields a typical overestimate by a factor of 1.2. To accurately determine the masses of cores from the millimeter continuum requires temperature determinations for every core. After this correction, the dominant contribution to the uncertainties in the core masses is the dust opacity at these wavelengths.

The column density of ammonia is typical for cores in Perseus (Jijina et al. 1999). However, the sensitive observations also find some spectra that imply $N\left(\mathrm{NH}_{3}\right)<10^{13.5} \mathrm{~cm}^{-2}$, making these detections among the lowest column densities of ammonia yet found. The low column density detections are all associated with the IC348 region of the cloud. The $\mathrm{C}_{2} \mathrm{~S}$ column densities also appear typical of dense cores (Suzuki et al. 1992) but are subject to uncertainty based on unknown temperatures and excitation conditions.
The velocity dispersions of the $\mathrm{C}_{2} \mathrm{~S}$ lines are comparable to those of the ammonia lines, and the $\mathrm{C}_{2} \mathrm{~S}$ lines show a slight, systematic offset in velocity from the ammonia complex. This offset is likely due to uncertainties in the assumed rest frequency of the $\mathrm{C}_{2} \mathrm{~S}$ line. The mean offset is $16 \mathrm{~m} \mathrm{~s}^{-1}$ (weighting by the inverse variance of the measurements) and would be consistent with zero for a rest frequency of $\nu_{\mathrm{CCS}}=22.344032(1) \mathrm{GHz}$. The difference is within the uncertainties of the assumed frequency.

We conclude this section by noting several spectra that define the extent of the property distributions or are otherwise notable. Plots of the spectra are available in the online-only edition (Figure 6a-6ff).

Typical Spectrum - NH3SRC 15 is the "most typical" ammonia spectrum from Perseus with nearly average values of all the properties shown in Figure 5. For NH3SRC 15, 
$T_{\text {kin }}=11.2 \mathrm{~K}, \sigma_{v}=0.19 \mathrm{~km} \mathrm{~s}^{-1}, \tau_{1,1}=3.7$, and $N\left(\mathrm{NH}_{3}\right)=$ $3.1 \times 10^{14} \mathrm{~cm}^{-2}$.

Temperature Range - NH3SRC 18 has the lowest, welldetermined temperature of the observed sources $\left(T_{k i n}=\right.$ $9.05 \mathrm{~K})$ and NH3SRC 116 has the highest temperature $\left(T_{k i n}=\right.$ $26 \mathrm{~K})$.

Column Density - NH3SRC 144 has the lowest column density detected in our survey $\left(5.5 \times 10^{12} \mathrm{~cm}^{-2}\right)$ and NH3SRC 17 has the highest column density $\left(1.3 \times 10^{15} \mathrm{~cm}^{-2}\right)$.

Line Brightness - NH3SRC 54 is the faintest source in $\mathrm{NH}_{3}(1,1)$ emission included as a detection $\left(W_{m b}=\right.$ $0.2 \mathrm{~K} \mathrm{~km} \mathrm{~s}^{-1}$ ) and NH3SRC 12 is the strongest $\left(20.0 \mathrm{~K} \mathrm{~km} \mathrm{~s}^{-1}\right)$. In the $(2,2)$ line, NH3SRC 19 is the weakest $\left(W_{m b}=0.03 \mathrm{~K} \mathrm{~km} \mathrm{~s}^{-1}\right)$ and NH3SRC 68 is the strongest $\left(W_{m b}=2.27 \mathrm{~K} \mathrm{~km} \mathrm{~s}^{-1}\right)$. In the $\mathrm{C}_{2} \mathrm{~S}$ line, NH3SRC 21 is the weakest detection $\left(W_{m b}=0.07 \mathrm{~K} \mathrm{~km} \mathrm{~s}^{-1}\right)$ while NH3SRC 42 is the strongest $\left(W_{m b}=0.67 \mathrm{~K} \mathrm{~km} \mathrm{~s}^{-1}\right)$.

Line Width - The narrowest line width source we detect is NH3SRC 128 with a line width of $0.079 \mathrm{~km} \mathrm{~s}^{-1}$. The largest line width we reliably detect is $0.23 \mathrm{~km} \mathrm{~s}^{-1}$ in NH3SRC 109. However, many of the fits yield larger results such as NH3SRC 71 where the measured line width is $0.72 \mathrm{~km} \mathrm{~s}^{-1}$ though the fit is unreliable. In addition many spectra show odd structure in their line profiles including wings (NH3SRCs $70,127)$ and plateaus (NH3SRC 75) in addition to the multicomponent structure discussed previously ( $\$ 3.4)$.

\section{SUMMARY}

We have searched for $\mathrm{NH}_{3}(1,1), \mathrm{NH}_{3}(1,1), \mathrm{C}_{2} \mathrm{~S}\left(2_{1} \rightarrow 1_{0}\right)$ emission along 193 lines of sight towards the Perseus molecular cloud. The lines of sight were selected based on positions that were detected in (sub)millimeter emission or had large dust column densities implied by far infrared (FIR) emission. We detect ammonia emission along $162(84 \%)$ of the lines of sight and $\mathrm{C}_{2} \mathrm{~S}$ along $96(51 \%)$ of the lines of sight. We estimate the physical properties of the gas by fitting a model emission profile to all spectral lines simultaneously. The emission is modeled as a uniform slab of gas that completely fills the beam, has a Gaussian intrinsic line width, and a single excitation temperature for all lines. Where appropriate, we refined the model to account for low optical depths, incomplete coupling to the GBT beam and multiple velocity components along the line of sight.

Nearly all (98\%) bright, (sub)millimeter cores have strong ammonia emission associated with them and the exceptions appear to be artifacts in the submillimeter map based on examining the original BOLOCAM data. In addition, we detected emission towards 23 sources selected based on
FIR emission that implies large dust column densities and low temperatures. Twenty-one objects are not seen in the (sub)millimeter, suggesting that the submillimeter emission is not a perfect tracer of the dense gas (the remaining two sources are outside the bounds of the continuum surveys). However, the FIR-based ammonia detections have lower line intensities than (sub)millimeter-bright source, as well as lower optical depths and larger line widths. It remains to be shown whether this could be an evolutionary effect or whether the (sub)millimeter-weak sources simply trace isolated pockets of gas not associated with the dense cores traced by the dust continuum.

We find that the ammonia implies dense gas temperatures in Perseus are predominantly cold $\left(T_{k} \sim 11 \mathrm{~K}\right)$. Ammonia column densities are typical for cores presented in the literature $\left(N_{\mathrm{NH} 3} \sim 10^{14.5} \mathrm{~cm}^{-2}\right.$, Jijina et al. 1999) though we also find several lines-of-sight with very low ammonia column densities $\left(N_{\mathrm{NH} 3} \lesssim 10^{13.5} \mathrm{~cm}^{-2}\right)$ associated with the IC 348 region.

Forthcoming work will examine the properties of these objects in more detail including comparison with the (sub)millimeter emission, protostellar content, and the velocity structure of the dense core population.

The Green Bank Telescope is operated by the National Radio Astronomy Observatory. The National Radio Astronomy Observatory is a facility of the National Science Foundation operated under cooperative agreement by Associated Universities, Inc. We are grateful for the assistance of Ron Maddalena and Frank Ghigo in preparation for the observations and to the GBT operators who executed the observations. We acknowledge the indispensable assistance and advice of Scott Schnee, Doug Johnstone, Melissa Enoch and Helen Kirk in the planning of and preparation for the observations, particularly for the use of their continuum maps. ER's work is supported by an NSF Astronomy and Astrophysics Postdoctoral Fellowship (AST-0502605). JEP and JBF are supported by a generous grant from the NRAO Student Observing Support Program (GSSP06-0015). JEP is supported by the National Science Foundation through grant \#AF002 from the Association of Universities for Research in Astronomy, Inc., under NSF cooperative agreement AST-9613615 and by Fundación Andes under project No. C-13442. This material is based upon work supported by the National Science Foundation under Grant No. AST-0407172. PC acknowledges supported by the Italian Ministry of Reserach and University within a PRIN project.

Facilities: GBT (K-band/ACS)

\section{REFERENCES}

Bachiller, R. \& Cernicharo, J. 1986, A\&A, 168, 262

Bachiller, R., Guilloteau, S., \& Kahane, C. 1987, A\&A, 173, 324

Cernis, K. 1993, Baltic Astronomy, 2, 214

De Vries, C. H. \& Myers, P. C. 2005, ApJ, 620, 800

Di Francesco, J., Evans, N. J., Caselli, P., Myers, P. C., Shirley, Y., Aikawa, A., \& Tafalla, M. 2006, ArXiv Astrophysics e-prints

Enoch, M. L., Young, K. E., Glenn, J., Evans, N. J., Golwala, S., Sargent, A. I., Harvey, P., Aguirre, J., Goldin, A., Haig, D., Huard, T. L., Lange, A., Laurent, G., Maloney, P., Mauskopf, P., Rossinot, P., \& Sayers, J. 2006, ApJ, 638, 293

Flower, D. R., Pineau Des Forêts, G., \& Walmsley, C. M. 2006, A\&A, 456, 215

Hatchell, J., Fuller, G. A., Richer, J. S., Harries, T. J., \& Ladd, E. F. 2007, A\&A, 468, 1009
Hatchell, J., Richer, J. S., Fuller, G. A., Qualtrough, C. J., Ladd, E. F., \& Chandler, C. J. 2005, A\&A, 440, 151

Ho, P. T. P., Barrett, A. H., Myers, P. C., Matsakis, D. N., Chui, M. F., Townes, C. H., Cheung, A. C., \& Yngvesson, K. S. 1979, ApJ, 234, 912 Ho, P. T. P. \& Townes, C. H. 1983, ARA\&A, 21, 239

Jijina, J., Myers, P. C., \& Adams, F. C. 1999, ApJS, 125, 161

Jørgensen, J. K., Harvey, P. M., Evans, II, N. J., Huard, T. L., Allen, L. E.,

Porras, A., Blake, G. A., Bourke, T. L., Chapman, N., Cieza, L., Koerner, D. W., Lai, S.-P., Mundy, L. G., Myers, P. C., Padgett, D. L., Rebull, L., Sargent, A. I., Spiesman, W., Stapelfeldt, K. R., van Dishoeck, E. F., Wahhaj, Z., \& Young, K. E. 2006, ApJ, 645, 1246

Jørgensen, J. K., Johnstone, D., Kirk, H., \& Myers, P. C. 2007, ApJ, 656, 293

Juan, J., Bachiller, R., Koempe, C., \& Martin-Pintado, J. 1993, A\&A, 270, 432

Kirk, H., Johnstone, D., \& Di Francesco, J. 2006, ApJ, 646, 1009 

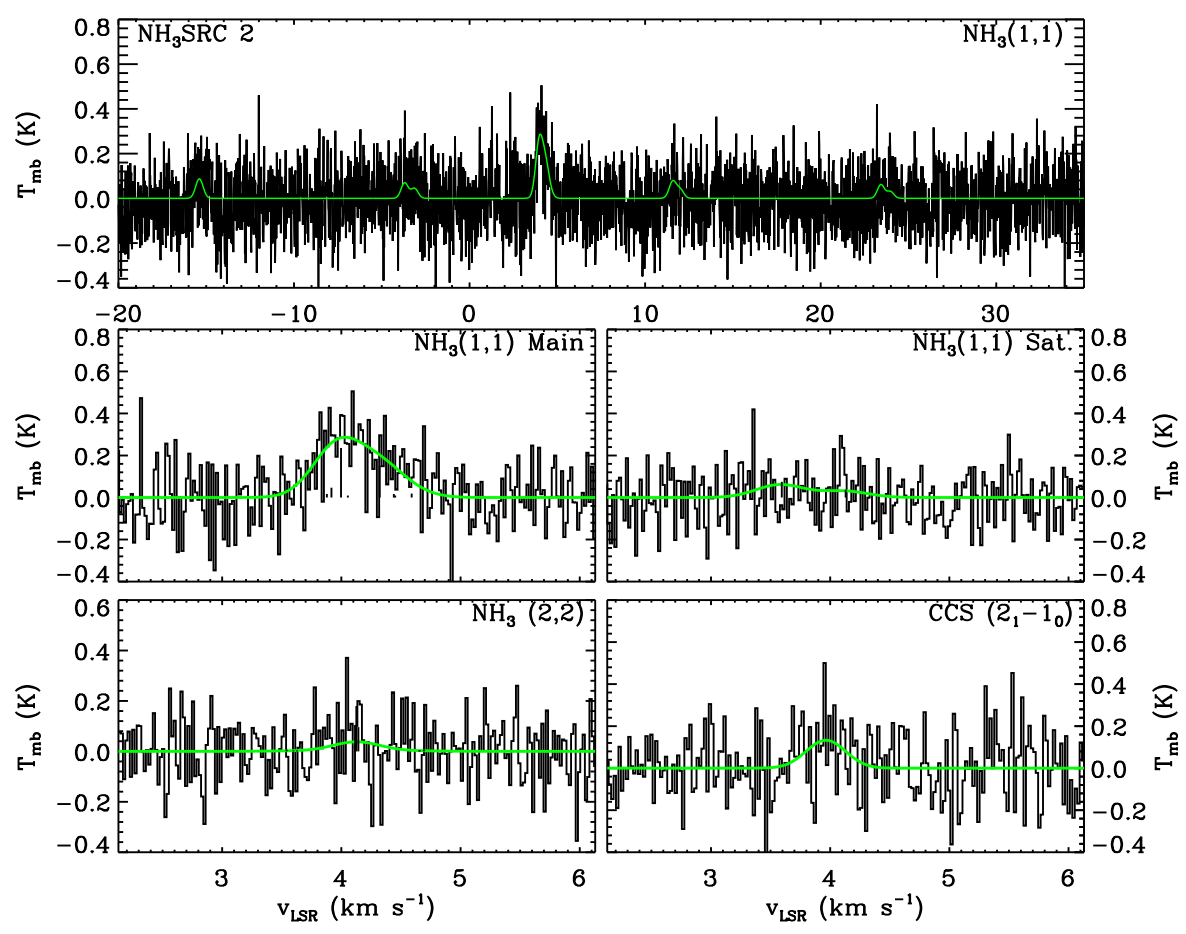

FIG. 6A.- (top) The full $\mathrm{NH}_{3}(1,1)$ spectrum of $\mathrm{NH}_{3} \mathrm{SRC} 1$. The spectrum is shown in black, the best fitting model described in $\$ 3$ is shown in green. Where discernible, additional velocity components are plotted in blue. (middle,left) The central component of the $\mathrm{NH}_{3}(1,1)$ line and best fitting model. The

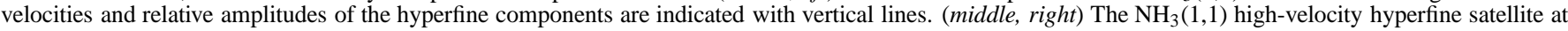
$\Delta v=19.85 \mathrm{~km} \mathrm{~s}^{-1}$ and best fitting model. Hyperfine components are indicated with vertical lines. (bottom, left) The main component of $\mathrm{NH}_{3}(2,2)$ line and best fitting model. Hyperfine components are indicated with vertical lines. (bottom, right) The $\mathrm{C}_{2} \mathrm{~S}\left(2_{1} \rightarrow 1_{0}\right)$ line and best fitting model.

Ladd, E. F., Myers, P. C., \& Goodman, A. A. 1994, ApJ, 433, 117

Langer, W. D., Velusamy, T., Kuiper, T. B. H., Levin, S., Olsen, E., \& Migenes, V. 1995, ApJ, 453, 293

Lovas, F. J. \& Dragoset, R. 2003, Recommended rest frequencies for observed interstellar molecular microwave transitions (Washington: National Bureau of Standards (NBS), 2003, Rev. ed.)

Mauersberger, R., Wilson, T. L., \& Henkel, C. 1988, A\&A, 201, 123

Motte, F., Andre, P., \& Neri, R. 1998, A\&A, 336, 150

Ohishi, M. \& Kaifu, N. 1998, in Chemistry and Physics of Molecules and Grains in Space. Faraday Discussions No. 109, 205-+

Pickett, H. M., Poynter, R. L., Cohen, E. A., Delitsky, M. L., Pearson, J. C., \& Muller, H. S. P. 1998, J. Quant. Spectrosc. \& Rad. Transfer, 60, 883

Press, W. H., Teukolsky, S. A., Vetterling, W. T., \& Flannery, B. P. 1992, Numerical recipes in $\mathrm{C}$. The art of scientific computing (Cambridge: University Press, |c1992, 2nd ed.)

Rebull, L. M., Stapelfeldt, K. R., Evans, II, N. J., Joergensen, J. K., Harvey, P. M., Brooke, T. Y., Bourke, T. L., Padgett, D. L., Chapman, N. L., Lai, S. ., Spiesmann, W. J., Noreiga-Crespo, A., Merin, B., Huard, T., Allen, L. E., Blake, G. A., Jarrett, T., Koerner, D. W., Mundy, L. G., Myers, P. C., Sargent, A. I., van Dishoeck, E. F., Wahhaj, Z., \& Young, K. E. 2007, ArXiv Astrophysics e-prints
Ridge, N. A., Di Francesco, J., Kirk, H., Li, D., Goodman, A. A., Alves, J. F., Arce, H. G., Borkin, M. A., Caselli, P., Foster, J. B., Heyer, M. H., Johnstone, D., Kosslyn, D. A., Lombardi, M., Pineda, J. E., Schnee, S. L., \& Tafalla, M. 2006, AJ, 131, 2921

Rohlfs, K. \& Wilson, T. L. 2004, Tools of radio astronomy (Tools of radio astronomy, 4th rev. and enl. ed., by K. Rohlfs and T.L. Wilson. Berlin: Springer, 2004)

Schnee, S., Li, J., \& Goodman, A. A. in preparation, ApJ

Suzuki, H., Yamamoto, S., Ohishi, M., Kaifu, N., Ishikawa, S.-I., Hirahara, Y., \& Takano, S. 1992, ApJ, 392, 551

Swade, D. A. 1989, ApJ, 345, 828

Swift, J. J., Welch, W. J., \& Di Francesco, J. 2005, ApJ, 620, 823

Tafalla, M., Myers, P. C., Caselli, P., \& Walmsley, C. M. 2004, A\&A, 416, 191

Testi, L. \& Sargent, A. I. 1998, ApJ, 508, L91

Wilson, T. L. \& Rood, R. 1994, ARA\&A, 32, 191

Yamamoto, S., Saito, S., Kawaguchi, K., Chikada, Y., Suzuki, H., Kaifu, N., Ishikawa, S.-I., \& Ohishi, M. 1990, ApJ, 361, 318 


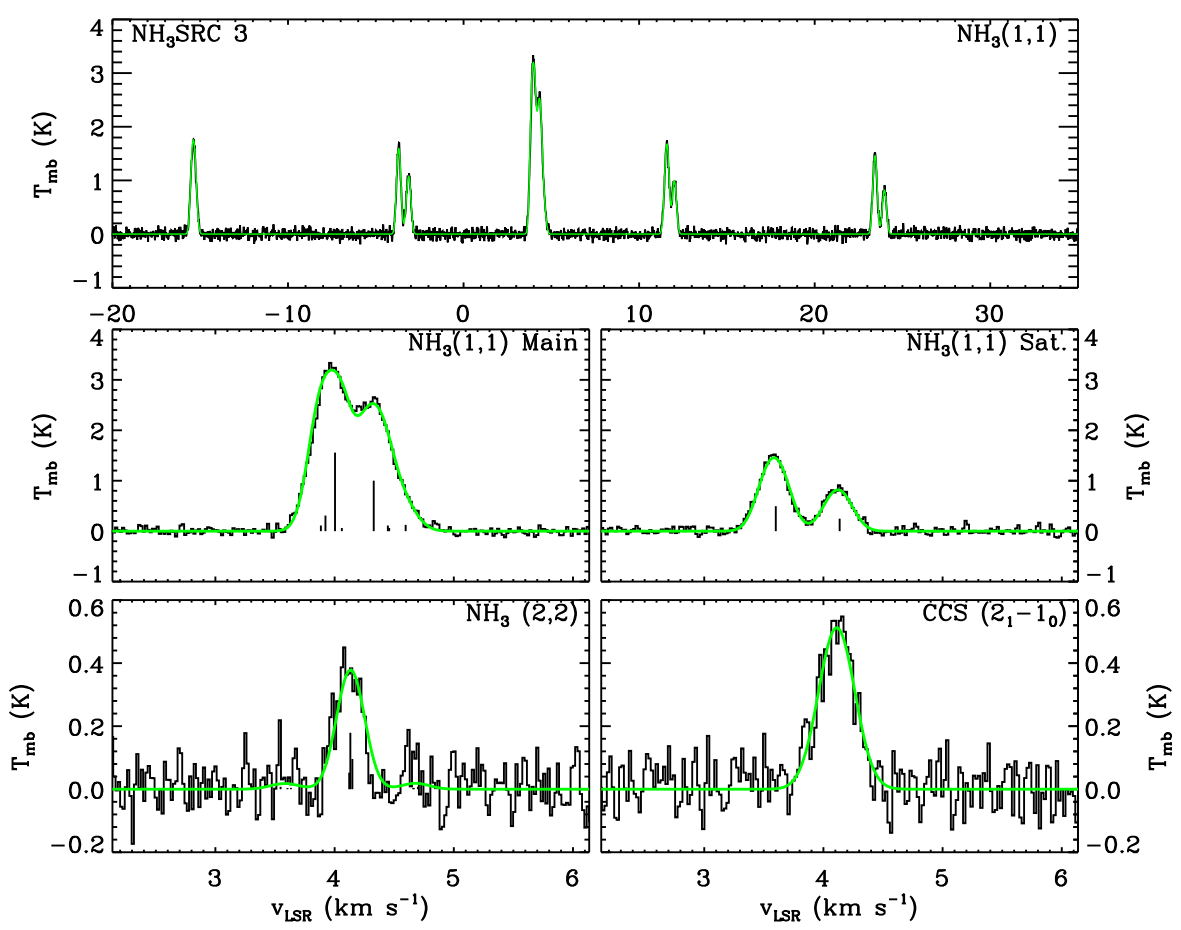

FIG. 6B.- As Figure 6a but for $\mathrm{NH}_{3} \mathrm{SRC} 3$.

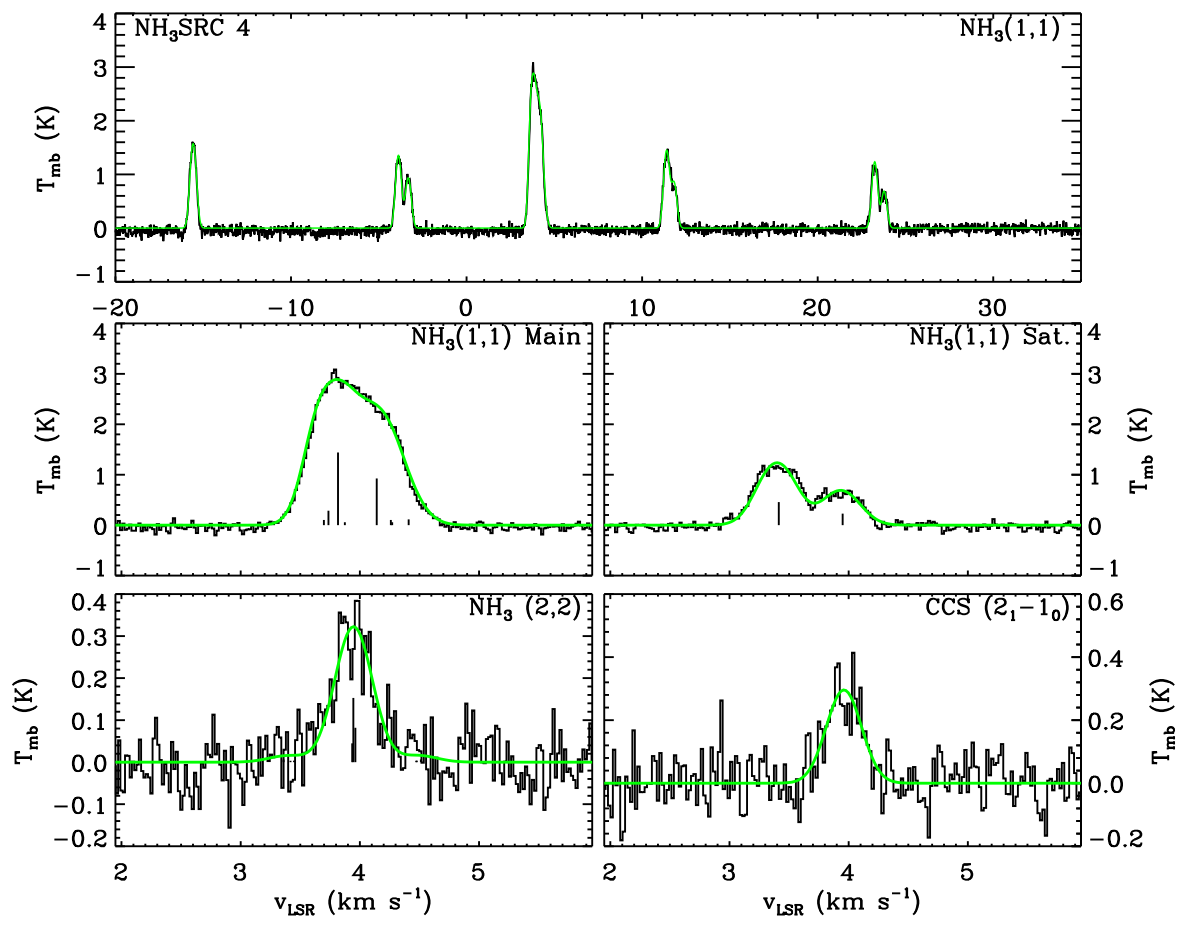

FIG. 6C.- As Figure 6a but for $\mathrm{NH}_{3} \mathrm{SRC} 4$. 

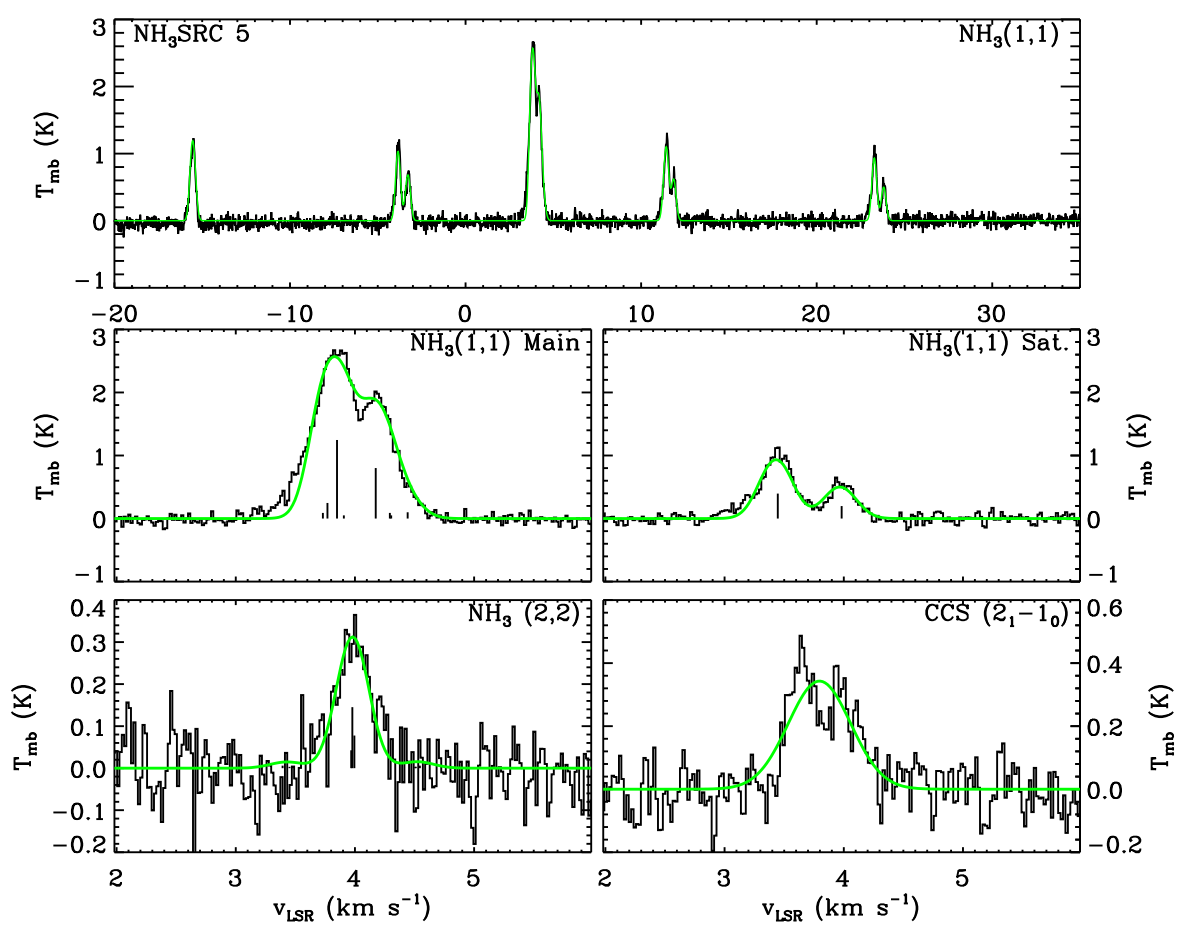

FIG. 6D.- As Figure 6a but for $\mathrm{NH}_{3} \mathrm{SRC} 5$. 
TABLE 2

SUMMARY OF OBSERVATIONS

\begin{tabular}{|c|c|c|c|c|c|c|c|c|c|c|}
\hline $\begin{array}{c}\text { NH3SRC } \\
\text { (1) }\end{array}$ & $\begin{array}{l}\text { Origin } \\
\text { (2) }\end{array}$ & $\begin{array}{c}\text { Region } \\
\text { (3) }\end{array}$ & $\begin{array}{c}\text { Position } \\
\left(\alpha_{2000}, \delta_{2000}\right) \\
(4)\end{array}$ & $\begin{array}{c}\text { Bolocam } \\
\text { Name } \\
(5)\end{array}$ & $\begin{array}{l}\text { SCUBA } \\
\text { Name } \\
(6)\end{array}$ & $\begin{array}{l}\text { Int. Time } \\
\text { (min.) } \\
(7)\end{array}$ & $\begin{array}{c}\sigma_{r m s} \\
(\mathrm{mK}) \\
(8)\end{array}$ & $\begin{array}{c}W\left[\mathrm{NH}_{3}(1,1)\right] \\
\left(\mathrm{K} \mathrm{km} \mathrm{s}^{-1}\right) \\
(9)\end{array}$ & $\begin{array}{c}W\left[\mathrm{NH}_{3}(2,2)\right] \\
\left(\mathrm{K} \mathrm{km} \mathrm{s}^{-1}\right) \\
(10)\end{array}$ & $\begin{array}{c}W\left[\mathrm{C}_{2} \mathrm{~S}\right] \\
\left(\mathrm{K} \mathrm{km} \mathrm{s}^{-1}\right) \\
(11)\end{array}$ \\
\hline 1 & $\mathrm{D}$ & & $03: 22: 18.9+30: 53: 14$ & $\cdots$ & N/A & 5 & 120 & $0.07(8)$ & $0.03(3)$ & $0.00(4)$ \\
\hline 2 & $\mathrm{D}$ & L1455/L1448 & $03: 25: 00.3+30: 44: 10$ & $\cdots$ & $\cdots$ & 5 & 128 & $0.46(9)$ & $0.02(4)$ & $0.05(5)$ \\
\hline 3 & $\mathrm{~B}$ & L1455/L1448 & $03: 25: 07.8+30: 24: 22$ & 1 & $\cdots$ & 15 & 63 & $5.25(4)$ & $0.15(2)$ & $0.22(2)$ \\
\hline 4 & $\mathrm{~B}$ & L1455/L1448 & $03: 25: 09.7+30: 23: 53$ & 2 & $\ldots$ & 15 & 60 & $5.87(4)$ & $0.12(2)$ & $0.09(2)$ \\
\hline 5 & $\mathrm{~B}$ & L1455/L1448 & $03: 25: 10.1+30: 44: 41$ & 3 & $\cdots$ & 15 & 63 & $4.08(4)$ & $0.10(2)$ & $0.23(2)$ \\
\hline 6 & $\mathrm{~B}$ & L1455/L1448 & $03: 25: 17.1+30: 18: 53$ & 4 & N/A & 20 & 54 & $2.21(4)$ & $0.04(1)$ & $0.36(2)$ \\
\hline 7 & $\mathrm{~B}$ & L1455/L1448 & $03: 25: 22.3+30: 45: 09$ & 5 & $032537+30451$ & 15 & 60 & $10.09(4)$ & $0.67(2)$ & $0.20(2)$ \\
\hline 8 & $\mathrm{~S}$ & L1455/L1448 & $03: 25: 26.2+30: 45: 05$ & $\ldots$ & $032543+30450$ & 10 & 87 & $12.36(6)$ & $0.79(2)$ & $0.34(3)$ \\
\hline 9 & B & L1455/L1448 & $03: 25: 26.9+30: 21: 53$ & 6 & N/A & 15 & 61 & 4.43(4) & $0.10(2)$ & $0.23(2)$ \\
\hline 10 & $\mathrm{D}$ & L1455/L1448 & $03: 25: 32.3+30: 46: 00$ & $\cdots$ & $\cdots$ & 10 & 74 & $2.93(5)$ & $0.10(2)$ & $0.06(3)$ \\
\hline 11 & $\mathrm{~B}$ & L1455/L1448 & $03: 25: 35.5+30: 13: 06$ & 7 & N/A & 15 & 58 & $0.94(4)$ & $0.04(2)$ & $0.36(2)$ \\
\hline 12 & B & L1455/L1448 & $03: 25: 36.2+30: 45: 11$ & 8 & $032560+30453$ & 20 & 54 & $19.97(4)$ & $1.65(1)$ & $0.24(2)$ \\
\hline 13 & $\mathrm{~B}$ & L1455/L1448 & $03: 25: 37.2+30: 09: 55$ & 9 & N/A & 20 & 44 & $0.61(3)$ & $0.04(1)$ & $0.12(2)$ \\
\hline 14 & $\mathrm{~B}$ & L1455/L1448 & $03: 25: 38.6+30: 43: 59$ & 10 & $032564+30440$ & 10 & 62 & $13.29(4)$ & $1.29(2)$ & $0.38(2)$ \\
\hline 15 & $\mathrm{~B}$ & L1455/L1448 & $03: 25: 46.1+30: 44: 11$ & 11 & $\ldots$ & 10 & 61 & $5.62(4)$ & $0.26(2)$ & $0.16(2)$ \\
\hline 16 & B & L1455/L1448 & $03: 25: 47.5+30: 12: 26$ & 12 & N/A & 20 & 41 & $0.71(3)$ & $0.02(1)$ & $0.36(2)$ \\
\hline 17 & $\mathrm{~B}$ & L1455/L1448 & $03: 25: 48.8+30: 42: 24$ & 13 & $032581+30423$ & 15 & 40 & $11.49(3)$ & $0.34(1)$ & $0.26(2)$ \\
\hline 18 & $\mathrm{~B}$ & L1455/L1448 & $03: 25: 50.6+30: 42: 02$ & 14 & $\ldots$ & 10 & 66 & $10.32(5)$ & $0.27(2)$ & $0.25(3)$ \\
\hline 19 & $\mathrm{~B}$ & L1455/L1448 & $03: 25: 55.1+30: 41: 26$ & 15 & $\cdots$ & 25 & 42 & $1.63(3)$ & $0.03(1)$ & $0.20(2)$ \\
\hline 20 & $\mathrm{~B}$ & L1455/L1448 & $03: 25: 56.4+30: 40: 43$ & 16 & $\cdots$ & 25 & 39 & $0.80(3)$ & $0.02(1)$ & $0.16(1)$ \\
\hline 21 & $\mathrm{~B}$ & L1455/L1448 & $03: 25: 58.5+30: 37: 14$ & 17 & $\ldots$ & 25 & 39 & $0.89(3)$ & $0.02(1)$ & $0.07(2)$ \\
\hline 22 & B & L1455/L1448 & $03: 26: 37.0+30: 15: 23$ & 18 & $032662+30153$ & 10 & 60 & $4.89(4)$ & $0.30(2)$ & $0.26(2)$ \\
\hline 23 & $\mathrm{D}$ & $\ldots$ & $03: 26: 39.7+31: 28: 21$ & N/A & N/A & 5 & 94 & $0.30(7)$ & $0.02(3)$ & $0.07(4)$ \\
\hline 24 & $\mathrm{~B}$ & L1455/L1448 & $03: 27: 02.1+30: 15: 08$ & 19 & $\ldots$ & 15 & 44 & $1.74(3)$ & $0.05(1)$ & $0.21(2)$ \\
\hline 25 & $\mathrm{D}$ & $\ldots$ & $03: 27: 14.3+31: 32: 49$ & $\cdots$ & N/A & 5 & 138 & $0.7(1)$ & $0.06(4)$ & $-0.04(5)$ \\
\hline 26 & $\mathrm{D}$ & L1455/L1448 & $03: 27: 20.2+30: 04: 26$ & $\cdots$ & N/A & 10 & 77 & $0.95(5)$ & $0.04(2)$ & $0.00(3)$ \\
\hline 27 & $\mathrm{D}$ & L1455/L1448 & $03: 27: 20.5+30: 00: 42$ & N/A & N/A & 5 & 108 & $0.75(7)$ & $0.08(3)$ & $0.18(4)$ \\
\hline 28 & $\mathrm{D}$ & $\ldots$ & $03: 27: 26.4+29: 51: 08$ & N/A & N/A & 10 & 122 & $0.74(8)$ & $0.01(4)$ & $0.19(5)$ \\
\hline 29 & $\mathrm{~B}$ & L1455/L1448 & $03: 27: 28.9+30: 15: 04$ & 20 & $\ldots$ & 20 & 54 & $4.51(4)$ & $0.16(2)$ & $0.36(2)$ \\
\hline 30 & $\mathrm{~L}$ & L1455/L1448 & $03: 27: 34.4+30: 09: 22$ & $\cdots$ & $\ldots$ & 5 & 87 & $1.54(6)$ & $0.02(2)$ & $0.13(3)$ \\
\hline 31 & $\mathrm{~B}$ & L1455/L1448 & $03: 27: 37.7+30: 14: 00$ & 21 & $032763+30139$ & 30 & 38 & $6.04(3)$ & $0.31(1)$ & $0.15(1)$ \\
\hline 32 & $\mathrm{~B}$ & L1455/L1448 & $03: 27: 39.3+30: 12: 59$ & 22 & $032765+30130$ & 20 & 45 & $10.56(3)$ & $0.85(1)$ & $0.20(2)$ \\
\hline 33 & $\mathrm{~S}$ & L1455/L1448 & $03: 27: 40.0+30: 12: 13$ & $\cdots$ & $032766+30122$ & 5 & 136 & $11.96(9)$ & $0.55(4)$ & $0.30(6)$ \\
\hline 34 & $\mathrm{~B}$ & L1455/L1448 & $03: 27: 41.9+30: 12: 30$ & 23 & $032771+30125$ & 20 & 41 & $10.78(3)$ & $0.61(1)$ & $0.25(2)$ \\
\hline 35 & $\mathrm{~B}$ & L1455/L1448 & $03: 27: 47.9+30: 12: 02$ & 24 & $032780+30121$ & 30 & 39 & $6.36(3)$ & $0.38(1)$ & $0.16(2)$ \\
\hline 36 & $\mathrm{~L}$ & L1455/L1448 & $03: 27: 55.9+30: 06: 18$ & $\cdots$ & N/A & 5 & 84 & $4.96(6)$ & $0.12(2)$ & $0.17(3)$ \\
\hline 37 & $\mathrm{~L}$ & L1455/L1448 & 03:28:00.7 +30:08:20 & $\cdots$ & $\cdots$ & 5 & 132 & 4.01(9) & $0.18(4)$ & $0.25(5)$ \\
\hline 38 & $\mathrm{~L}$ & L1455/L1448 & $03: 28: 05.5+30: 06: 19$ & $\cdots$ & N/A & 5 & 80 & $5.00(6)$ & $0.14(2)$ & $0.14(3)$ \\
\hline 39 & $\mathrm{~W}$ & NGC1333 & $03: 28: 30.0+30: 55: 29$ & $\cdots$ & $\ldots$ & 5 & 131 & $0.09(9)$ & $-0.01(4)$ & $0.05(6)$ \\
\hline 40 & B & NGC1333 & $03: 28: 32.2+31: 11: 09$ & 25 & $\cdots$ & 15 & 38 & $5.36(3)$ & $0.25(1)$ & $0.05(2)$ \\
\hline 41 & $\mathrm{~B}$ & NGC1333 & $03: 28: 32.4+31: 04: 43$ & 26 & $\cdots$ & 10 & 54 & $6.36(4)$ & $0.29(2)$ & $0.10(2)$ \\
\hline 42 & $\mathrm{~B}$ & L1455/L1448 & $03: 28: 33.4+30: 19: 35$ & 27 & $\cdots$ & 15 & 68 & $2.24(5)$ & $0.12(2)$ & $0.66(2)$ \\
\hline 43 & $\mathrm{~B}$ & NGC1333 & $03: 28: 34.1+31: 07: 01$ & 28 & $\ldots$ & 15 & 54 & $3.01(4)$ & $0.12(2)$ & $0.06(2)$ \\
\hline 44 & $\mathrm{~B}$ & NGC1333 & $03: 28: 36.3+31: 13: 27$ & 29 & $032861+31134$ & 15 & 41 & $5.26(3)$ & $0.34(1)$ & $0.02(2)$ \\
\hline 45 & $\mathrm{~W}$ & NGC1333 & $03: 28: 36.8+31: 00: 14$ & $\cdots$ & $\ldots$ & 5 & 142 & $0.00(1)$ & $0.00(4)$ & $0.03(6)$ \\
\hline 46 & $\mathrm{~B}$ & NGC1333 & $03: 28: 39.1+31: 06: 00$ & 30 & $032865+31060$ & 10 & 57 & $8.85(4)$ & $0.42(2)$ & $0.24(2)$ \\
\hline 47 & $\mathrm{~S}$ & NGC1333 & $03: 28: 39.5+31: 18: 35$ & $\cdots$ & $032865+31185$ & 41 & 34 & $12.60(2)$ & $0.847(9)$ & $0.18(1)$ \\
\hline 48 & $\mathrm{~S}$ & NGC1333 & $03: 28: 40.3+31: 17: 56$ & 31 & $032866+31179$ & 5 & 170 & $16.2(1)$ & $1.09(4)$ & $0.10(7)$ \\
\hline 49 & $\mathrm{~B}$ & L1455/L1448 & $03: 28: 41.7+30: 31: 12$ & 32 & $\ldots$ & 15 & 41 & $0.94(3)$ & $-0.02(1)$ & $0.37(2)$ \\
\hline 50 & $\mathrm{~B}$ & NGC1333 & $03: 28: 42.6+31: 06: 13$ & 33 & $\cdots$ & 10 & 57 & $8.26(4)$ & $0.37(2)$ & $0.14(2)$ \\
\hline 51 & $\mathrm{~B}$ & NGC1333 & $03: 28: 46.0+31: 15: 19$ & 34 & $\cdots$ & 10 & 65 & $8.78(4)$ & $0.42(2)$ & $0.08(3)$ \\
\hline 52 & $\mathrm{~B}$ & NGC1333 & $03: 28: 48.5+31: 16: 03$ & 35 & $\cdots$ & 10 & 66 & $7.22(5)$ & $0.39(2)$ & $0.06(3)$ \\
\hline 53 & $\mathrm{~B}$ & L1455/L1448 & $03: 28: 48.8+30: 43: 25$ & 36 & $\ldots$ & 20 & 40 & $0.63(3)$ & $0.04(1)$ & $0.10(2)$ \\
\hline 54 & $\mathrm{D}$ & NGC1333 & $03: 28: 49.6+31: 30: 01$ & $\ldots$ & $\cdots$ & 10 & 75 & $0.20(5)$ & $-0.02(2)$ & $0.00(3)$ \\
\hline 55 & $\mathrm{D}$ & L1455/L1448 & $03: 28: 51.4+30: 32: 58$ & $\cdots$ & $\cdots$ & 10 & 113 & $0.77(8)$ & $0.02(3)$ & $0.05(4)$ \\
\hline 56 & $\mathrm{~B}$ & NGC1333 & $03: 28: 52.2+31: 18: 08$ & 37 & $\ldots$ & 10 & 76 & $6.12(5)$ & $0.52(2)$ & $0.04(3)$ \\
\hline 57 & $\mathrm{D}$ & NGC1333 & $03: 28: 55.2+31: 20: 26$ & $\cdots$ & $\ldots$ & 10 & 82 & $2.16(6)$ & $0.24(2)$ & $-0.04(3)$ \\
\hline 58 & B & NGC1333 & $03: 28: 55.3+31: 14: 33$ & 38 & $032891+31145$ & 10 & 63 & $11.13(4)$ & $1.44(2)$ & $0.17(3)$ \\
\hline 59 & $\mathrm{~B}$ & NGC1333 & $03: 28: 55.4+31: 19: 19$ & 39 & $\ldots$ & 10 & 71 & $8.00(5)$ & $0.87(2)$ & $0.02(3)$ \\
\hline 60 & $\mathrm{D}$ & L1455/L1448 & $03: 28: 56.2+30: 03: 42$ & N/A & N/A & 10 & 97 & $0.51(7)$ & $0.02(3)$ & $0.13(4)$ \\
\hline 61 & $\mathrm{D}$ & NGC1333 & $03: 28: 57.5+31: 23: 06$ & $\cdots$ & $\cdots$ & 10 & 80 & $0.66(6)$ & $0.07(2)$ & $0.03(3)$ \\
\hline 62 & $\mathrm{D}$ & L1455/L1448 & $03: 28: 58.1+30: 45: 12$ & $\cdots$ & $\cdots$ & 10 & 104 & $0.53(7)$ & $0.04(3)$ & $0.01(4)$ \\
\hline 63 & $\mathrm{D}$ & NGC1333 & $03: 28: 58.6+31: 09: 10$ & $\cdots$ & $\ldots$ & 5 & 115 & $0.56(8)$ & $0.03(3)$ & $-0.08(4)$ \\
\hline 64 & $\mathrm{~B}$ & NGC1333 & $03: 28: 59.6+31: 21: 38$ & 40 & $032899+31215$ & 10 & 69 & $8.47(5)$ & $0.85(2)$ & $0.08(3)$ \\
\hline 65 & $\mathrm{~B}$ & NGC1333 & $03: 29: 00.6+31: 11: 59$ & 41 & $032900+31119$ & 10 & 61 & 7.87(4) & $0.54(2)$ & $0.03(2)$ \\
\hline 66 & $\mathrm{~B}$ & NGC1333 & $03: 29: 01.4+31: 20: 34$ & 42 & $032901+31204$ & 10 & 86 & $11.46(6)$ & $1.55(2)$ & $0.03(3)$ \\
\hline 67 & $\mathrm{~S}$ & NGC1333 & $03: 29: 03.2+31: 15: 59$ & 43 & $032905+31159$ & 10 & 113 & $12.93(8)$ & $1.71(3)$ & $0.05(4)$ \\
\hline 68 & $\mathrm{~S}$ & NGC1333 & $03: 29: 03.4+31: 14: 58$ & $\ldots$ & $032905+31149$ & 10 & 80 & $16.77(6)$ & $2.27(2)$ & $0.09(3)$ \\
\hline 69 & $\mathrm{~B}$ & NGC1333 & $03: 29: 04.5+31: 18: 43$ & 44 & $\ldots$ & 10 & 69 & $6.41(5)$ & $0.54(2)$ & $0.06(3)$ \\
\hline 70 & $\mathrm{~S}$ & NGC1333 & $03: 29: 06.9+31: 15: 44$ & $\cdots$ & $032910+31156$ & 15 & 101 & $13.10(7)$ & $1.45(3)$ & $0.17(4)$ \\
\hline 71 & $\mathrm{~S}$ & NGC1333 & $03: 29: 07.5+31: 21: 54$ & $\cdots$ & $032912+31218$ & 10 & 57 & $0.75(4)$ & $0.19(2)$ & $-0.02(2)$ \\
\hline 72 & $\mathrm{~B}$ & NGC1333 & $03: 29: 07.8+31: 17: 19$ & 45 & $032911+31173$ & 10 & 72 & $7.35(5)$ & $0.55(2)$ & $0.03(3)$ \\
\hline 73 & $\mathrm{~S}$ & NGC1333 & $03: 29: 08.9+31: 15: 12$ & 46 & $032914+31152$ & 10 & 56 & $18.40(4)$ & $1.31(2)$ & $0.20(2)$ \\
\hline 74 & $\mathrm{D}$ & L1455/L1448 & $03: 29: 09.6+30: 21: 18$ & $\ldots$ & $\ldots$ & 5 & 112 & $0.21(8)$ & $0.00(3)$ & $0.00(4)$ \\
\hline
\end{tabular}


TABLE $2-$ Continued

\begin{tabular}{|c|c|c|c|c|c|c|c|c|c|c|}
\hline $\begin{array}{c}\text { NH3SRC } \\
\text { (1) }\end{array}$ & $\begin{array}{l}\text { Origin } \\
\text { (2) }\end{array}$ & $\begin{array}{l}\text { Region } \\
\text { (3) }\end{array}$ & $\begin{array}{c}\text { Position } \\
\left(\alpha_{2000}, \delta_{2000}\right) \\
(4)\end{array}$ & $\begin{array}{l}\text { Bolocam } \\
\text { Name } \\
\text { (5) }\end{array}$ & $\begin{array}{l}\text { SCUBA } \\
\text { Name } \\
(6)\end{array}$ & $\begin{array}{l}\text { Int. Time } \\
\text { (min.) } \\
(7)\end{array}$ & $\begin{array}{c}\sigma_{r m s} \\
(\mathrm{mK}) \\
(8)\end{array}$ & $\begin{array}{c}W\left[\mathrm{NH}_{3}(1,1)\right] \\
\left(\mathrm{K} \mathrm{km} \mathrm{s}^{-1}\right) \\
(9)\end{array}$ & $\begin{array}{c}W\left[\mathrm{NH}_{3}(2,2)\right] \\
\left(\mathrm{K} \mathrm{km} \mathrm{s}^{-1}\right) \\
(10)\end{array}$ & $\begin{array}{c}W\left[\mathrm{C}_{2} \mathrm{~S}\right] \\
\left(\mathrm{K} \mathrm{km} \mathrm{s}^{-1}\right) \\
(11)\end{array}$ \\
\hline 75 & $\mathrm{~S}$ & NGC1333 & $03: 29: 10.3+31: 13: 35$ & 48 & $032916+31135$ & 15 & 58 & $11.28(4)$ & $1.25(2)$ & $0.13(2)$ \\
\hline 77 & B & NGC1333 & $03: 29: 11.4+31: 18: 26$ & 49 & $032917+31184$ & 10 & 65 & $10.10(5)$ & $1.02(2)$ & $0.06(3)$ \\
\hline 78 & $\mathrm{~s}$ & NGC1333 & $03: 29: 11.4+31: 13: 07$ & $\ldots$ & $032919+31131$ & 10 & 264 & $7.4(2)$ & $0.67(7)$ & $0.1(1)$ \\
\hline 79 & B & NGC1333 & $03: 29: 14.9+31: 20: 27$ & 50 & $032925+31205$ & 15 & 52 & $2.08(4)$ & $0.20(1)$ & $-0.01(2)$ \\
\hline 80 & B & NGC1333 & $03: 29: 17.0+31: 12: 26$ & 51 & & 30 & 54 & $6.02(4)$ & $0.28(1)$ & $0.02(2)$ \\
\hline 84 & B & NGC1333 & $03: 29: 19.2+31: 23: 28$ & 54 & $\ldots$ & 20 & 72 & $2.44(5)$ & $0.18(2)$ & $-0.09(3)$ \\
\hline 85 & $\mathrm{D}$ & NGC1333 & $03: 29: 20.5+31: 19: 30$ & $\ldots$ & $\ldots$ & 10 & 73 & $0.55(5)$ & $0.05(2)$ & $0.04(3)$ \\
\hline 86 & $\mathrm{~B}$ & NGC1333 & $03: 29: 22.5+31: 36: 24$ & 56 & $\ldots$ & 20 & 67 & $2.93(5)$ & $0.04(2)$ & $0.01(3)$ \\
\hline 87 & B & NGC1333 & $03: 29: 22.9+31: 33: 16$ & 57 & $032939+31333$ & 25 & 65 & $6.81(5)$ & $0.31(2)$ & $0.02(3)$ \\
\hline 88 & B & NGC1333 & $03: 29: 25.8+31: 28: 17$ & 58 & $032942+31283$ & 10 & 63 & $7.67(4)$ & $0.28(2)$ & $0.05(3)$ \\
\hline 89 & $\mathrm{~B}$ & NGC1333 & $03: 29: 51.5+31: 39: 12$ & 59 & $032986+31391$ & 20 & 70 & $6.80(5)$ & $0.29(2)$ & $0.08(3)$ \\
\hline 90 & $\mathrm{D}$ & NGC1333 & $03: 30: 13.6+31: 44: 38$ & $\ldots$ & N/A & 10 & 121 & $0.36(8)$ & $0.03(3)$ & $-0.07(4)$ \\
\hline 96 & B & $\mathrm{B} 1-\mathrm{W}$ & $03: 30: 45.6+30: 52: 36$ & 63 & $\ldots$ & 20 & 60 & $2.70(4)$ & $0.07(2)$ & $0.32(2)$ \\
\hline 97 & $\mathrm{~B}$ & $\mathrm{~B} 1-\mathrm{W}$ & $03: 30: 50.5+30: 49: 17$ & 64 & $\ldots$ & 23 & 62 & $2.47(4)$ & $0.04(2)$ & $0.13(2)$ \\
\hline 98 & $\mathrm{D}$ & $\mathrm{B} 1-\mathrm{W}$ & $03: 31: 14.4+30: 44: 03$ & & $\ldots$ & 10 & 127 & $1.15(9)$ & $0.17(4)$ & $0.01(5)$ \\
\hline 99 & $\mathrm{~B}$ & $\mathrm{~B} 1-\mathrm{W}$ & $03: 31: 20.0+30: 45: 30$ & 65 & $033134+30454$ & 15 & 40 & $8.86(3)$ & $0.53(1)$ & $0.03(2)$ \\
\hline 100 & $\mathrm{D}$ & $\ldots$ & $03: 31: 58.4+30: 02: 04$ & N/A & N/A & 10 & 96 & $0.12(7)$ & $0.04(3)$ & $0.01(3)$ \\
\hline 101 & $\mathrm{D}$ & B1 & $03: 32: 10.1+31: 19: 54$ & $\ldots$ & $\ldots$ & 5 & 130 & $0.62(9)$ & $-0.02(4)$ & $0.03(5)$ \\
\hline 102 & $\mathrm{~W}$ & B1 & $03: 32: 17.5+30: 53: 58$ & $\ldots$ & $\ldots$ & 10 & 145 & $0.7(1)$ & $0.03(4)$ & $0.21(6)$ \\
\hline 103 & B & B1 & $03: 32: 17.5+30: 49: 49$ & 66 & $033229+30497$ & 15 & 63 & $11.28(4)$ & $0.72(2)$ & $0.24(3)$ \\
\hline 104 & B & B1 & $03: 32: 26.9+30: 59: 11$ & 67 & $\ldots$ & 15 & 40 & $6.54(3)$ & $0.28(1)$ & $0.64(1)$ \\
\hline 105 & B & B1 & $03: 32: 28.1+31: 02: 19$ & 68 & $\ldots$ & 15 & 55 & $3.66(4)$ & $0.11(2)$ & $0.14(2)$ \\
\hline 106 & $\mathrm{~W}$ & B1 & $03: 32: 28.6+30: 53: 51$ & $\ldots$ & $\ldots$ & 10 & 155 & $0.6(1)$ & $-0.03(4)$ & $0.05(6)$ \\
\hline 107 & $\mathrm{~B}$ & B1 & $03: 32: 39.3+30: 57: 29$ & 69 & $\ldots$ & 32 & 43 & $1.42(3)$ & $0.03(1)$ & $0.18(2)$ \\
\hline 108 & $\mathrm{~B}$ & B1 & $03: 32: 44.1+31: 00: 01$ & 70 & $\ldots$ & 10 & 60 & $8.72(4)$ & $0.35(2)$ & $0.28(2)$ \\
\hline 109 & B & B1 & $03: 32: 51.3+31: 01: 48$ & 71 & $\ldots$ & 20 & 42 & $1.77(3)$ & $0.07(1)$ & $0.22(2)$ \\
\hline 110 & $\mathrm{D}$ & B1 & $03: 32: 54.8+31: 19: 23$ & $\ldots$ & $\ldots$ & 10 & 75 & $0.76(5)$ & $0.04(2)$ & $0.06(3)$ \\
\hline 121 & B & B1 & $03: 33: 17.9+31: 09: 30$ & 80 & $033329+31095$ & 20 & 40 & $16.40(3)$ & $1.23(1)$ & $0.41(2)$ \\
\hline 122 & $\mathrm{D}$ & B1 & $03: 33: 19.8+31: 22: 41$ & $\ldots$ & $\ldots$ & 10 & 75 & $0.70(5)$ & $0.03(2)$ & $0.01(3)$ \\
\hline 123 & B & B1 & $03: 33: 20.5+31: 07: 37$ & 81 & $033335+31075$ & 15 & 45 & $18.75(3)$ & $1.25(1)$ & $0.43(2)$ \\
\hline 124 & B & B1 & $03: 33: 25.2+31: 05: 35$ & 82 & $\ldots$ & 10 & 56 & $6.69(4)$ & $0.27(2)$ & $0.14(2)$ \\
\hline 125 & B & B1 & $03: 33: 25.4+31: 20: 05$ & 83 & $\ldots$ & 20 & 47 & $2.05(3)$ & $0.14(1)$ & $0.21(2)$ \\
\hline 126 & B & B1 & $03: 33: 27.1+31: 06: 56$ & 84 & $\ldots$ & 15 & 57 & $4.13(4)$ & $0.23(2)$ & $0.27(2)$ \\
\hline 127 & $\mathrm{~B}$ & B1 & $03: 33: 31.8+31: 20: 02$ & 85 & $\ldots$ & 20 & 56 & $3.58(4)$ & $0.14(2)$ & $0.07(2)$ \\
\hline 128 & B & B1 & $03: 33: 51.2+31: 12: 38$ & 86 & $\ldots$ & 20 & 41 & $1.16(3)$ & $0.03(1)$ & $0.01(2)$ \\
\hline 129 & $\mathrm{D}$ & B1 & $03: 33: 52.5+31: 22: 37$ & $\cdots$ & $\ldots$ & 10 & 120 & $0.99(8)$ & $0.08(3)$ & $-0.02(5)$ \\
\hline 130 & $\mathrm{D}$ & B1-E & $03: 34: 59.9+31: 15: 17$ & $\ldots$ & $\ldots$ & 7 & 182 & $0.3(1)$ & $-0.07(5)$ & $-0.10(7)$ \\
\hline 131 & $\mathrm{D}$ & B1-E & $03: 35: 02.2+30: 55: 06$ & $\ldots$ & $\ldots$ & 5 & 117 & $-0.14(8)$ & $0.00(3)$ & $0.00(4)$ \\
\hline 132 & $\mathrm{~B}$ & B1-E & $03: 35: 21.5+31: 06: 56$ & 87 & $\ldots$ & 15 & 59 & $0.73(4)$ & $0.06(2)$ & $0.11(3)$ \\
\hline 133 & $\mathrm{D}$ & B1-E & $03: 35: 42.6+31: 09: 53$ & $\ldots$ & $\ldots$ & 5 & 116 & $0.30(9)$ & $-0.01(4)$ & $0.04(5)$ \\
\hline 134 & $\mathrm{~W}$ & B1-E & $03: 35: 56.5+31: 15: 21$ & $\ldots$ & $\ldots$ & 5 & 143 & $0.4(1)$ & $0.00(4)$ & $0.03(6)$ \\
\hline 135 & $\mathrm{D}$ & B1-E & $03: 36: 44.6+31: 13: 42$ & $\ldots$ & $\ldots$ & 5 & 128 & $0.19(9)$ & $0.04(4)$ & $0.00(5)$ \\
\hline 136 & $\mathrm{~W}$ & $\ldots$ & $03: 37: 07.5+31: 33: 25$ & $\ldots$ & N/A & 5 & 146 & $-0.2(1)$ & $-0.03(4)$ & $-0.14(5)$ \\
\hline 137 & $\mathrm{~W}$ & $\ldots$ & $03: 37: 14.5+31: 24: 16$ & $\ldots$ & $\ldots$ & 5 & 146 & $0.0(1)$ & $0.06(4)$ & $0.03(6)$ \\
\hline 138 & $\mathrm{D}$ & $\ldots$ & $03: 38: 09.0+30: 46: 28$ & $\ldots$ & N/A & 5 & 119 & $0.21(8)$ & $0.02(3)$ & $-0.01(4)$ \\
\hline 139 & $\mathrm{~W}$ & $\ldots$ & $03: 38: 15.1+31: 19: 45$ & $\ldots$ & $\ldots$ & 10 & 53 & $0.43(4)$ & $0.10(2)$ & $0.02(2)$ \\
\hline 140 & $\mathrm{D}$ & $\ldots$ & $03: 39: 00.6+30: 41: 07$ & N/A & N/A & 5 & 120 & $0.56(8)$ & $0.02(3)$ & $-0.03(4)$ \\
\hline 141 & B & IC348 & $03: 40: 14.5+32: 01: 30$ & 88 & N/A & 10 & 140 & $1.2(1)$ & $0.08(4)$ & $0.05(6)$ \\
\hline 142 & B & IC348 & $03: 40: 49.5+31: 48: 35$ & 89 & $\ldots$ & 10 & 61 & $2.17(4)$ & $0.14(2)$ & $0.14(2)$ \\
\hline 143 & B & IC348 & $03: 41: 09.3+31: 44: 33$ & 90 & $\ldots$ & 20 & 71 & $0.02(5)$ & $-0.06(2)$ & $-0.04(3)$ \\
\hline 144 & B & IC348 & $03: 41: 19.9+31: 47: 28$ & 91 & $\ldots$ & 10 & 51 & $0.47(4)$ & $0.06(1)$ & $0.00(2)$ \\
\hline 145 & B & IC348 & $03: 41: 40.2+31: 58: 05$ & 92 & $\ldots$ & 5 & 156 & $4.8(1)$ & $0.19(4)$ & $0.01(6)$ \\
\hline 146 & B & IC348 & $03: 41: 45.2+31: 48: 09$ & 93 & $\ldots$ & 10 & 51 & $0.59(4)$ & $0.07(1)$ & $0.01(2)$ \\
\hline 147 & $\mathrm{~B}$ & IC348 & $03: 41: 46.0+31: 57: 22$ & 94 & $\ldots$ & 5 & 70 & $5.90(5)$ & $0.24(2)$ & $0.01(2)$ \\
\hline 148 & $\mathrm{~W}$ & IC348 & $03: 41: 58.3+31: 58: 36$ & $\cdots$ & $\ldots$ & 5 & 223 & $1.3(2)$ & $0.11(6)$ & $0.14(9)$ \\
\hline 149 & $\mathrm{~W}$ & IC348 & $03: 42: 09.0+31: 46: 50$ & $\ldots$ & $\ldots$ & 5 & 204 & $0.3(1)$ & $-0.01(6)$ & $0.03(8)$ \\
\hline
\end{tabular}


TABLE 2 - Continued

\begin{tabular}{|c|c|c|c|c|c|c|c|c|c|c|}
\hline $\begin{array}{c}\text { NH3SRC } \\
\text { (1) }\end{array}$ & $\begin{array}{l}\text { Origin } \\
(2)\end{array}$ & $\begin{array}{c}\text { Region } \\
\text { (3) }\end{array}$ & $\begin{array}{c}\text { Position } \\
\left(\alpha_{2000}, \delta_{2000}\right) \\
(4)\end{array}$ & $\begin{array}{l}\text { Bolocam } \\
\text { Name } \\
(5)\end{array}$ & $\begin{array}{l}\text { SCUBA } \\
\text { Name } \\
(6)\end{array}$ & $\begin{array}{l}\text { Int. Time } \\
\text { (min.) } \\
(7)\end{array}$ & $\begin{array}{c}\sigma_{r m s} \\
(\mathrm{mK}) \\
(8)\end{array}$ & $\begin{array}{c}W\left[\mathrm{NH}_{3}(1,1)\right] \\
\left(\mathrm{K} \mathrm{km} \mathrm{s}^{-1}\right) \\
(9)\end{array}$ & $\begin{array}{c}W\left[\mathrm{NH}_{3}(2,2)\right] \\
\left(\mathrm{K} \mathrm{km} \mathrm{s}^{-1}\right) \\
(10)\end{array}$ & $\begin{array}{c}W\left[\mathrm{C}_{2} \mathrm{~S}\right] \\
\left(\mathrm{K} \mathrm{km} \mathrm{s}^{-1}\right) \\
(11)\end{array}$ \\
\hline 150 & B & IC348 & $03: 42: 20.3+31: 44: 51$ & 95 & $\cdots$ & 15 & 51 & $0.43(4)$ & $0.04(1)$ & $0.00(2)$ \\
\hline 151 & $\mathrm{~W}$ & IC348 & $03: 42: 24.0+31: 45: 43$ & $\cdots$ & $\cdots$ & 15 & 56 & $0.59(4)$ & $0.03(2)$ & $0.04(2)$ \\
\hline 152 & B & IC348 & $03: 42: 47.2+31: 58: 41$ & 96 & $\cdots$ & 5 & 135 & $0.95(9)$ & $0.17(4)$ & $0.16(6)$ \\
\hline 153 & B & IC 348 & $03: 42: 52.5+31: 58: 11$ & 97 & $\ldots$ & 5 & 168 & $0.9(1)$ & $0.10(5)$ & $0.03(7)$ \\
\hline 154 & B & IC348 & $03: 42: 57.3+31: 57: 48$ & 98 & $\cdots$ & 10 & 150 & $1.0(1)$ & $0.12(4)$ & $-0.07(6)$ \\
\hline 155 & $\mathrm{~W}$ & IC348 & $03: 43: 29.6+31: 55: 22$ & $\cdots$ & $\ldots$ & 5 & 204 & $0.5(1)$ & $0.01(6)$ & $0.03(8)$ \\
\hline 156 & $\mathrm{~B}$ & IC 348 & $03: 43: 38.1+32: 03: 10$ & 99 & $034363+32032$ & 5 & 150 & $4.3(1)$ & $0.35(4)$ & $0.06(6)$ \\
\hline 157 & $\mathrm{~S}$ & IC348 & $03: 43: 44.0+32: 02: 52$ & $\cdots$ & $034373+32028$ & 15 & 44 & $3.89(3)$ & $0.27(1)$ & $0.06(2)$ \\
\hline 158 & B & IC348 & $03: 43: 45.5+32: 01: 44$ & 101 & $\ldots$ & 15 & 52 & $1.50(4)$ & $0.13(1)$ & $0.06(2)$ \\
\hline 159 & $\mathrm{~S}$ & IC348 & $03: 43: 45.8+32: 03: 11$ & $\cdots$ & $034376+32031$ & 10 & 54 & $5.63(4)$ & $0.35(2)$ & $0.02(2)$ \\
\hline 160 & B & IC348 & $03: 43: 50.5+32: 03: 17$ & 102 & $034385+32033$ & 5 & 151 & 8.3(1) & $0.51(4)$ & $0.09(6)$ \\
\hline 161 & $\mathrm{~B}$ & IC 348 & $03: 43: 56.0+32: 00: 45$ & 103 & $034394+32008$ & 5 & 153 & $9.5(1)$ & $0.70(4)$ & $0.15(6)$ \\
\hline 162 & B & IC348 & $03: 43: 57.3+32: 03: 04$ & 104 & $034395+32030$ & 5 & 150 & $3.6(1)$ & $0.35(4)$ & $0.08(6)$ \\
\hline 163 & B & IC 348 & $03: 43: 57.8+32: 04: 06$ & 105 & $034396+32040$ & 15 & 57 & $3.62(4)$ & $0.36(2)$ & $0.06(2)$ \\
\hline 164 & B & IC348 & $03: 44: 01.7+32: 02: 02$ & 106 & $034402+32020$ & 5 & 135 & $4.42(9)$ & $0.37(4)$ & $0.08(6)$ \\
\hline 165 & B & IC348 & $03: 44: 02.2+32: 02: 32$ & 107 & $034404+32025$ & 10 & 55 & $6.74(4)$ & $0.36(2)$ & $0.01(2)$ \\
\hline 166 & $\mathrm{~B}$ & IC 348 & $03: 44: 02.3+32: 04: 56$ & 108 & $\ldots$ & 15 & 54 & $0.77(4)$ & $0.15(2)$ & $0.00(2)$ \\
\hline 167 & $\mathrm{D}$ & IC348 & $03: 44: 04.6+31: 58: 07$ & $\cdots$ & $\cdots$ & 10 & 69 & $0.80(5)$ & $0.10(2)$ & $0.01(3)$ \\
\hline 168 & $\mathrm{~B}$ & IC348 & $03: 44: 05.1+32: 00: 28$ & 109 & $\ldots$ & 10 & 139 & $1.2(1)$ & $0.06(4)$ & $0.00(6)$ \\
\hline 169 & B & IC 348 & $03: 44: 05.3+32: 02: 05$ & 110 & $\cdots$ & 10 & 138 & $5.4(1)$ & $0.27(4)$ & $0.03(6)$ \\
\hline 170 & B & IC348 & $03: 44: 14.6+31: 57: 59$ & 111 & $\cdots$ & 5 & 145 & 4.1(1) & $0.09(4)$ & $0.08(6)$ \\
\hline 171 & $\mathrm{~B}$ & IC 348 & $03: 44: 14.7+32: 09: 11$ & 112 & $\ldots$ & 15 & 57 & $1.94(4)$ & $0.13(2)$ & $-0.02(2)$ \\
\hline 172 & $\mathrm{~W}$ & IC 348 & $03: 44: 18.4+32: 06: 36$ & $\cdots$ & $\cdots$ & 5 & 179 & $0.0(1)$ & $0.00(5)$ & $0.00(7)$ \\
\hline 173 & $\mathrm{~B}$ & IC348 & $03: 44: 22.6+31: 59: 24$ & 113 & $\ldots$ & 5 & 144 & $6.4(1)$ & $0.23(4)$ & $0.09(6)$ \\
\hline 174 & B & IC 348 & $03: 44: 22.6+32: 10: 00$ & 114 & $\cdots$ & 15 & 71 & $1.43(5)$ & $0.16(2)$ & $0.01(3)$ \\
\hline 175 & $\mathrm{D}$ & IC348 & $03: 44: 30.0+31: 59: 04$ & $\cdots$ & $\ldots$ & 5 & 123 & $0.45(9)$ & $0.10(4)$ & $-0.05(5)$ \\
\hline 176 & $\mathrm{~B}$ & IC 348 & $03: 44: 36.4+31: 58: 40$ & 115 & $034461+31587$ & 10 & 65 & $2.89(5)$ & $0.13(2)$ & $0.04(2)$ \\
\hline 177 & W & IC348 & $03: 44: 37.7+32: 08: 13$ & $\cdots$ & $\ldots$ & 5 & 187 & $-0.1(1)$ & $-0.06(6)$ & $-0.09(8)$ \\
\hline 178 & $\mathrm{~B}$ & IC 348 & $03: 44: 44.0+32: 01: 24$ & 116 & $034472+32015$ & 10 & 136 & $1.62(9)$ & $0.24(4)$ & $0.05(5)$ \\
\hline 179 & W & IC 348 & $03: 44: 46.2+32: 10: 50$ & $\cdots$ & $\ldots$ & 5 & 180 & $0.2(1)$ & $-0.03(5)$ & $-0.07(7)$ \\
\hline 180 & B & IC348 & $03: 44: 48.8+32: 00: 29$ & 117 & $\cdots$ & 5 & 138 & $3.2(1)$ & $0.13(4)$ & $-0.04(6)$ \\
\hline 181 & $\mathrm{~B}$ & IC 348 & $03: 44: 56.1+32: 00: 32$ & 118 & $\ldots$ & 15 & 52 & $1.48(4)$ & $0.07(1)$ & $0.04(2)$ \\
\hline 182 & $\mathrm{D}$ & IC348 & $03: 45: 10.7+32: 00: 38$ & $\cdots$ & $\cdots$ & 10 & 69 & $0.36(5)$ & $0.01(2)$ & $0.01(3)$ \\
\hline 183 & B & IC348 & $03: 45: 15.9+32: 04: 49$ & 119 & $\ldots$ & 5 & 144 & $3.5(1)$ & $0.14(4)$ & $-0.02(6)$ \\
\hline 184 & B & $\ldots$ & $03: 45: 48.0+32: 24: 13$ & 120 & N/A & 5 & 171 & $0.2(1)$ & $-0.01(5)$ & $0.02(7)$ \\
\hline 185 & $\mathrm{D}$ & B5 & $03: 46: 43.0+32: 41: 38$ & $\cdots$ & N/A & 5 & 123 & $0.33(9)$ & $-0.01(3)$ & $0.01(5)$ \\
\hline 186 & $\mathrm{~W}$ & B5 & $03: 47: 07.8+32: 42: 01$ & $\ldots$ & N/A & 5 & 145 & $0.1(1)$ & $0.00(4)$ & $0.03(6)$ \\
\hline 187 & W & B5 & $03: 47: 22.0+32: 45: 18$ & $\cdots$ & N/A & 5 & 138 & $0.2(1)$ & $0.04(4)$ & $-0.04(6)$ \\
\hline 188 & $\mathrm{~B}$ & B5 & $03: 47: 33.5+32: 50: 55$ & 121 & $\ldots$ & 10 & 55 & $2.37(4)$ & $0.10(1)$ & $0.11(2)$ \\
\hline 189 & $\mathrm{~S}$ & B5 & $03: 47: 38.6+32: 52: 19$ & $\cdots$ & $034764+32523$ & 5 & 154 & $6.0(1)$ & $0.21(4)$ & $0.20(6)$ \\
\hline 190 & $\mathrm{~L}$ & B5 & $03: 47: 39.7+32: 53: 57$ & $\cdots$ & $\ldots$ & 10 & 53 & $1.88(4)$ & $0.09(2)$ & $0.36(2)$ \\
\hline 191 & $\mathrm{D}$ & B5 & $03: 47: 39.8+32: 53: 34$ & $\ldots$ & $\ldots$ & 15 & 77 & $2.69(5)$ & $0.08(2)$ & $0.29(3)$ \\
\hline 192 & $\mathrm{~S}$ & B5 & $03: 47: 41.4+32: 51: 48$ & 122 & $034769+32517$ & 5 & 171 & $6.8(1)$ & $0.44(4)$ & $0.06(6)$ \\
\hline 193 & W & B5 & $03: 48: 28.1+32: 50: 15$ & $\cdots$ & N/A & 5 & 148 & $-0.3(1)$ & $0.04(4)$ & $-0.05(6)$ \\
\hline
\end{tabular}

NotE. - (1) Running Source Number. (2) Origin of Source: B: BOLOCAM Core from Enoch et al. (2006), S:SCUBA Core form Kirk et al. (2006), D: FIR Dust emission from Schnee et al. (in preparation), L: Literature sources in Jijina et al. (1999). (3) Designation as defined in Figure 1 (4) Position Observed. (5) Name of object in the Enoch et al. (2006) catalog. "N/A" is listed if the position is outside the boundaries of the BOLOCAM survey. (6) Name of object in the Kirk et al. (2006) catalog. "N/A" is listed if the position is outside the boundaries of the SCUBA survey. (7) Total integration time on source. (8) Noise level in the $\mathrm{NH}_{3}(1,1)$ spectrum on the $T_{m b}$ scale. (9)-(11) Integrated intensity of the observed lines on the $T_{m b}$ scale. 
TABLE 3

DERIVED PHysiCAL PROPERTIES

\begin{tabular}{|c|c|c|c|c|c|c|c|c|c|c|c|c|}
\hline NH3SRC & $\begin{array}{c}V_{L S R} \\
\left(\mathrm{~km} \mathrm{~s}^{-1}\right) \\
(2)\end{array}$ & $\begin{array}{c}\sigma_{v} \\
\left(\mathrm{~km} \mathrm{~s}^{-1}\right) \\
(3)\end{array}$ & $\begin{array}{c}T_{k} \\
(\mathrm{~K}) \\
(4)\end{array}$ & $\begin{array}{c}N_{\mathrm{NH} 3}{ }^{\mathrm{a}} \\
10^{13} \mathrm{~cm}^{-2} \\
(5)\end{array}$ & $\begin{array}{c}N_{\mathrm{CCS}^{\mathrm{a}}} \\
10^{12} \mathrm{~cm}^{-2} \\
(6)\end{array}$ & $\begin{array}{l}\tau_{1} \\
(7)\end{array}$ & $\begin{array}{l}T_{x}^{\mathrm{a}} \\
(\mathrm{K}) \\
(8)\end{array}$ & $\begin{array}{l}\eta_{f}^{\mathrm{a}} \\
(9)\end{array}$ & $\begin{array}{c}T_{\mathrm{CCS}}{ }^{\mathrm{a}} \\
(\mathrm{K}) \\
(10)\end{array}$ & $\begin{array}{c}\sigma_{\mathrm{CCS}} \\
\left(\mathrm{km} \mathrm{s}^{-1}\right) \\
(11)\end{array}$ & $\begin{array}{c}V_{o f f} \\
\left(\mathrm{~km} \mathrm{~s}^{-1}\right) \\
(12)\end{array}$ & $\begin{array}{l}\widetilde{\chi^{2}} \\
(13)\end{array}$ \\
\hline 2 & $4.13(5)$ & $<0.20(6)$ & $<26$ & $>0.6$ & $\ldots$ & \multicolumn{2}{|c|}{$0.8(2)$} & $\ldots$ & $\ldots$ & $\ldots$ & $\ldots$ & 1.0 \\
\hline 3 & $4.136(1)$ & $0.115(1)$ & $9.2(2)$ & 46.(2) & $4.0(4)$ & $6.6(2)$ & $6.47(7)$ & 0.58 & $0.51(4)$ & $0.15(1)$ & $0.02(1)$ & 0.9 \\
\hline 4 & $3.949(2)$ & $0.155(2)$ & $9.2(2)$ & 53.(2) & 2.3(4) & $6.1(2)$ & $6.14(6)$ & 0.52 & $0.29(4)$ & $0.15(2)$ & $0.06(2)$ & 1.1 \\
\hline 5 & $3.982(2)$ & $0.135(2)$ & $10.0(2)$ & 26.(2) & $5.2(8)$ & $3.8(2)$ & $6.5(2)$ & 0.52 & $0.34(3)$ & $0.26(3)$ & $-0.14(3)$ & 1.4 \\
\hline 6 & $4.030(3)$ & $0.134(3)$ & $10.3(4)$ & 12.(2) & $8.1(6)$ & $1.8(3)$ & $6.4(5)$ & 0.48 & $0.67(3)$ & $0.20(1)$ & $0.09(1)$ & 1.0 \\
\hline 7 & $4.135(1)$ & $0.168(1)$ & $12.06(8)$ & 54.(1) & $5.1(7)$ & 7.4(1) & $7.45(4)$ & 0.50 & $0.30(3)$ & $0.23(3)$ & $0.05(3)$ & 1.2 \\
\hline 8 & $4.090(1)$ & $0.155(1)$ & $11.21(9)$ & 84.(2) & 7.(1) & $10.9(2)$ & 7.61(4) & 0.57 & $0.53(5)$ & $0.19(2)$ & $0.09(2)$ & 1.0 \\
\hline 9 & $4.563(2)$ & $0.124(2)$ & $9.1(2)$ & 39.(3) & $3.7(5)$ & $5.5(2)$ & $6.04(9)$ & 0.52 & $0.44(4)$ & $0.16(2)$ & $-0.05(2)$ & 1.0 \\
\hline 10 & 4.295(9) & $0.29(1)$ & 11.1(4) & 28.(4) & $\ldots$ & $3.3(4)$ & 4.3(1) & 0.18 & $\ldots$ & $\ldots$ & $\ldots$ & 1.0 \\
\hline 11 & $4.236(8)$ & $0.143(8)$ & $<13$ & $>5$ & $<12$ & $1.2(7)$ & 5.(1) & 0.27 & $0.86(3)$ & $0.163(8)$ & $-0.01(1)$ & 0.9 \\
\hline $12^{\mathrm{b}}$ & $4.523(2)$ & $<0.373(2)$ & $12.61(5)$ & 91.(2) & $7.2(8)$ & $5.19(7)$ & $8.55(4)$ & 0.59 & $0.22(2)$ & $0.42(4)$ & $-0.05(4)$ & 2.7 \\
\hline 13 & $4.06(2)$ & $<0.24(2)$ & $<15$ & $>7$ & $<4.3$ & $1.5(7)$ & $3.5(3)$ & 0.12 & $0.24(2)$ & $0.19(2)$ & $0.06(3)$ & 0.9 \\
\hline 14 & $5.074(3)$ & $0.392(3)$ & $14.05(9)$ & $55 .(2)$ & 10.(1) & $3.60(9)$ & $7.83(7)$ & 0.45 & $0.46(3)$ & $0.24(2)$ & $-0.42(2)$ & 1.6 \\
\hline 15 & $4.648(3)$ & $0.194(3)$ & 11.2(2) & 31.(2) & $4.9(9)$ & $3.7(2)$ & $6.6(1)$ & 0.46 & $0.25(3)$ & $0.29(4)$ & $-0.06(4)$ & 1.0 \\
\hline 16 & $4.614(7)$ & $0.141(6)$ & $<13$ & $>1.3$ & $<12$ & \multicolumn{2}{|c|}{$1.85(8)$} & $\ldots$ & $1.07(3)$ & $0.132(4)$ & $0.026(8)$ & 1.0 \\
\hline $17^{\mathrm{b}}$ & $4.5058(7)$ & $0.1456(6)$ & $9.13(5)$ & 133.(2) & $4.8(4)$ & $14.7(2)$ & $6.71(2)$ & 0.62 & $0.30(2)$ & $0.32(2)$ & $-0.22(2)$ & 4.4 \\
\hline 18 & $4.539(1)$ & $0.153(1)$ & $9.0(1)$ & 119.(4) & $5.0(7)$ & $12.7(2)$ & $6.44(3)$ & 0.59 & $0.34(3)$ & $0.29(3)$ & $-0.10(3)$ & 3.3 \\
\hline 19 & $4.345(7)$ & $0.256(9)$ & $10.0(6)$ & 13.(4) & $4.7(5)$ & $1.4(3)$ & 4.7(4) & 0.27 & $0.37(2)$ & $0.22(2)$ & $-0.03(2)$ & 0.9 \\
\hline 20 & $4.17(1)$ & $<0.28(2)$ & $<14$ & $>1.3$ & $<6.0$ & \multicolumn{2}{|c|}{$1.02(5)$} & $\ldots$ & $0.43(2)$ & $0.16(1)$ & $0.06(2)$ & 0.9 \\
\hline 21 & $3.559(9)$ & $<0.21(1)$ & $<13.6$ & $>1.5$ & $<2.0$ & \multicolumn{2}{|c|}{$1.46(7)$} & $\ldots$ & $0.12(2)$ & $0.19(4)$ & $-0.02(4)$ & 0.9 \\
\hline 22 & $5.150(2)$ & $0.146(2)$ & $11.7(2)$ & 32.(1) & $6.4(7)$ & $6.3(2)$ & $5.63(6)$ & 0.32 & $0.57(4)$ & $0.16(1)$ & $0.04(1)$ & 1.0 \\
\hline 24 & $4.591(3)$ & $0.149(3)$ & $10.4(4)$ & 13.(2) & $5.5(6)$ & $2.2(3)$ & $5.1(3)$ & 0.32 & $0.53(3)$ & $0.17(1)$ & $0.03(1)$ & 0.8 \\
\hline 25 & $0.90(4)$ & $<0.20(5)$ & $\ldots$ & $\ldots$ & $\ldots$ & \multicolumn{2}{|c|}{$1.1(2)$} & $\ldots$ & $\ldots$ & $\ldots$ & $\ldots$ & 1.1 \\
\hline 26 & $5.32(3)$ & $<0.32(3)$ & $<18$ & $>7$ & $\ldots$ & 1.(1) & $3.7(6)$ & 0.10 & $\ldots$ & $\ldots$ & $\ldots$ & 1.0 \\
\hline 27 & $5.26(3)$ & $<0.22(4)$ & $<20$ & $>1.0$ & $<4$ & \multicolumn{2}{|c|}{$1.2(2)$} & $\ldots$ & $0.29(8)$ & $0.11(4)$ & $0.05(5)$ & 1.0 \\
\hline 28 & $5.62(1)$ & $<0.11(1)$ & $<18$ & $>3$ & $<9$ & 2.(2) & $5 .(2)$ & 0.29 & $0.9(1)$ & $0.08(1)$ & $0.09(2)$ & 1.0 \\
\hline 29 & $5.083(2)$ & $0.125(1)$ & $10.7(2)$ & 27.(1) & $6.6(4)$ & $4.8(2)$ & $6.4(1)$ & 0.46 & $1.00(4)$ & $0.105(5)$ & $-0.352(5)$ & 1.7 \\
\hline 30 & $5.22(1)$ & $<0.18(1)$ & $<13$ & $>9$ & $<3.7$ & $1.9(6)$ & $4.6(5)$ & 0.25 & $0.41(6)$ & $0.11(2)$ & $-0.10(2)$ & 0.9 \\
\hline 31.1 & $4.6(2)$ & $0.17(1)$ & $11.7(1)$ & 23.(2) & $2.7(3)$ & $3.749(2)$ & $\ldots$ & 0.43 & $0.120(5)$ & $0.32(3)$ & $0.10(6)$ & 4.1 \\
\hline 31.2 & $6.01(5)$ & $0.13(2)$ & $10.4(1)$ & 10.(1) & $1.8(3)$ & $1.739(2)$ & $\ldots$ & 0.24 & $0.175(6)$ & $0.17(3)$ & $0.02(2)$ & 4.1 \\
\hline 32 & $4.759(2)$ & $0.275(2)$ & $13.05(7)$ & 48.(1) & $5.7(8)$ & $4.40(8)$ & $7.46(5)$ & 0.46 & $0.22(2)$ & $0.31(3)$ & $0.09(3)$ & 2.3 \\
\hline 33 & $4.928(3)$ & $0.186(3)$ & $10.5(2)$ & 79.(4) & 7.(3) & $7.8(3)$ & $7.65(9)$ & 0.63 & $0.27(6)$ & $0.4(1)$ & $0.2(1)$ & 1.0 \\
\hline $34^{\mathrm{b}}$ & $4.9691(9)$ & $0.1690(8)$ & $11.37(6)$ & 54.(1) & $5.2(6)$ & $6.08(9)$ & $8.32(4)$ & 0.65 & $0.37(2)$ & $0.21(1)$ & $0.02(1)$ & 3.6 \\
\hline 35 & $4.949(2)$ & $0.211(2)$ & 11.9(1) & 32.(1) & $3.5(8)$ & $3.7(1)$ & $6.70(7)$ & 0.43 & $0.10(2)$ & $0.47(8)$ & $0.21(8)$ & 1.1 \\
\hline 36 & $4.706(1)$ & $0.083(1)$ & $9.1(2)$ & 48.(3) & $2.6(9)$ & $9.7(4)$ & $6.33(7)$ & 0.57 & $0.20(4)$ & $0.26(6)$ & $0.02(6)$ & 1.0 \\
\hline 37 & $4.861(3)$ & $0.115(3)$ & $10.3(4)$ & 27.(4) & 4.(1) & $5.0(5)$ & $6.2(2)$ & 0.45 & $0.40(9)$ & $0.17(4)$ & $0.01(4)$ & 0.9 \\
\hline 38 & $4.940(2)$ & $0.126(2)$ & $9.4(3)$ & 44.(3) & $2.8(7)$ & $6.6(3)$ & $5.99(9)$ & 0.49 & $0.28(5)$ & $0.18(3)$ & $0.22(3)$ & 0.9 \\
\hline 40 & 7.193(1) & $0.151(1)$ & $10.8(1)$ & 41.(1) & $1.6(4)$ & $6.9(2)$ & $5.62(4)$ & 0.36 & $0.14(2)$ & $0.19(4)$ & $0.06(4)$ & 1.0 \\
\hline 41 & $6.655(2)$ & $0.148(1)$ & $10.6(1)$ & 39.(1) & $2.7(7)$ & $5.4(2)$ & $6.83(7)$ & 0.52 & $0.13(3)$ & $0.33(7)$ & $-0.06(7)$ & 1.4 \\
\hline 42 & $5.471(4)$ & $0.136(4)$ & $10.7(3)$ & 18.(2) & 17.(1) & $3.8(4)$ & $4.9(2)$ & 0.27 & $1.85(3)$ & $0.150(3)$ & $0.125(5)$ & 1.3 \\
\hline 43 & $6.823(2)$ & $0.110(2)$ & $10.5(2)$ & 25.(2) & $1.2(6)$ & $6.0(3)$ & $5.21(8)$ & 0.32 & $0.19(5)$ & $0.10(3)$ & $0.06(3)$ & 1.0 \\
\hline 44 & $7.338(2)$ & $0.216(2)$ & $12.4(1)$ & 27.(1) & $1.5(5)$ & $3.6(1)$ & $6.13(7)$ & 0.35 & $0.11(3)$ & $0.18(5)$ & $0.06(5)$ & 1.3 \\
\hline 46 & 7.030(1) & $0.152(1)$ & $10.54(9)$ & 56.(2) & 4.1(7) & $7.0(1)$ & $7.35(5)$ & 0.59 & $0.33(3)$ & $0.20(2)$ & $0.07(2)$ & 1.3 \\
\hline 47 & $8.1840(7)$ & $0.1886(6)$ & $11.69(4)$ & $73.2(9)$ & $4.9(4)$ & $8.15(7)$ & $7.82(2)$ & 0.57 & $0.29(2)$ & $0.23(2)$ & $0.15(2)$ & 2.6 \\
\hline 48 & $7.985(3)$ & $0.192(2)$ & 11.7(1) & 91.(4) & $\ldots$ & $8.8(3)$ & 9.01(9) & 0.70 & $\ldots$ & $\ldots$ & $\ldots$ & 1.0 \\
\hline 49 & $5.311(9)$ & $<0.21(1)$ & $<13$ & $>1.8$ & $<12$ & 1.6 & & $\ldots$ & $0.99(3)$ & $0.159(5)$ & $0.05(1)$ & 1.1 \\
\hline 50 & $7.213(1)$ & $0.158(1)$ & $10.5(1)$ & $60 .(2)$ & $3.4(8)$ & $7.8(2)$ & $6.69(4)$ & 0.51 & $0.21(3)$ & $0.26(4)$ & $0.12(4)$ & 1.1 \\
\hline 51 & $8.113(2)$ & $0.194(2)$ & $10.8(1)$ & 49.(2) & $\ldots$ & $4.9(1)$ & $7.63(8)$ & 0.61 & $\ldots$ & $\ldots$ & $\ldots$ & 1.3 \\
\hline 52 & $8.056(1)$ & $0.124(1)$ & $11.3(1)$ & 40.(1) & $\ldots$ & $7.1(2)$ & 7.18(7) & 0.52 & $\ldots$ & $\ldots$ & $\ldots$ & 1.1 \\
\hline 53 & $5.84(2)$ & $<0.39(3)$ & $<17$ & $>0.94$ & $<4.3$ & 0.5 & & $\ldots$ & $0.38(3)$ & $0.10(1)$ & $-0.14(3)$ & 1.0 \\
\hline 54 & 7.91(4) & $<0.16(4)$ & $\ldots$ & $\ldots$ & $\ldots$ & 0.5 & & $\ldots$ & ... & $\ldots$ & $\ldots$ & 1.0 \\
\hline 55 & $5.39(3)$ & $<0.22(4)$ & $<19$ & $>11$ & $\ldots$ & 3.(2) & $3.2(2)$ & 0.08 & $\ldots$ & $\ldots$ & $\ldots$ & 1.0 \\
\hline 56 & $7.623(3)$ & $0.192(3)$ & $13.8(2)$ & 20.(2) & $\ldots$ & $2.5(2)$ & $8.4(3)$ & 0.51 & $\ldots$ & $\ldots$ & $\ldots$ & 1.0 \\
\hline 57 & $7.85(1)$ & $0.35(1)$ & $16.4(7)$ & $3.3(2)$ & $\ldots$ & 2.2 & & $\ldots$ & $\ldots$ & $\ldots$ & $\ldots$ & 0.9 \\
\hline 58 & $7.476(3)$ & $0.372(3)$ & $16.5(1)$ & 34.(2) & 6.(3) & $2.5(1)$ & $8.4(1)$ & 0.41 & $0.14(3)$ & $0.4(1)$ & $0.4(1)$ & 1.9 \\
\hline 59 & $7.778(2)$ & $0.172(2)$ & $14.5(1)$ & 28.(1) & $\ldots$ & 4.2(1) & 8.1(1) & 0.45 & $\ldots$ & $\ldots$ & $\ldots$ & 1.0 \\
\hline 60 & $5.73(3)$ & $<0.18(3)$ & $<19$ & $>7$ & $<7$ & 2.(2) & $3.3(3)$ & 0.09 & $0.36(6)$ & $0.15(3)$ & $0.04(4)$ & 0.9 \\
\hline 61 & 8.1(1) & $<0.7(1)$ & $<29$ & $>0.7$ & $\ldots$ & 0.2 & & $\ldots$ & $\ldots$ & .. & $\ldots$ & 1.0 \\
\hline 62 & $5.82(5)$ & $<0.26(6)$ & $\ldots$ & $\ldots$ & $\ldots$ & 0.7 & & $\ldots$ & $\ldots$ & $\ldots$ & $\ldots$ & 0.9 \\
\hline 63 & $7.8(1)$ & $<0.7(1)$ & $<26$ & $>0.9$ & $\ldots$ & 0.3 & & $\ldots$ & $\cdots$ & $\cdots$ & $\cdots$ & 1.0 \\
\hline 64 & $7.794(3)$ & $0.307(4)$ & $14.4(2)$ & 27.(2) & $\ldots$ & $2.2(1)$ & $8.6(2)$ & 0.50 & $\ldots$ & $\ldots$ & $\ldots$ & 0.9 \\
\hline 65 & $7.147(2)$ & $0.208(2)$ & $12.5(1)$ & 37.(1) & $1.9(7)$ & $4.5(1)$ & $6.98(8)$ & 0.43 & $0.15(4)$ & $0.17(5)$ & $0.13(5)$ & 1.2 \\
\hline 66 & $8.006(2)$ & $0.269(3)$ & $16.4(1)$ & 33.(1) & $\ldots$ & $2.8(1)$ & $10.0(2)$ & 0.53 & $\ldots$ & .. & .. & 0.9 \\
\hline 67 & $8.436(3)$ & $0.296(4)$ & $16.3(2)$ & 41.(2) & $\ldots$ & $3.6(2)$ & $8.7(2)$ & 0.44 & $\ldots$ & $\ldots$ & $\ldots$ & 0.9 \\
\hline $68^{b}$ & $7.433(5)$ & $<0.578(5)$ & $16.4(1)$ & 52.(3) & $\ldots$ & $2.3(1)$ & $9.0(2)$ & 0.46 & $\ldots$ & $\ldots$ & $\ldots$ & 1.6 \\
\hline 69 & $8.283(3)$ & $0.247(3)$ & $13.6(2)$ & 25.(1) & $\ldots$ & $2.7(1)$ & $7.3(2)$ & 0.42 & $\cdots$ & $\ldots$ & $\cdots$ & 0.9 \\
\hline 70 & $8.028(4)$ & $0.357(4)$ & $14.8(2)$ & 46.(2) & $\cdots$ & $3.2(1)$ & $8.5(2)$ & 0.48 & $\cdots$ & $\cdots$ & $\cdots$ & 1.3 \\
\hline 71 & $7.81(5)$ & $<0.72(5)$ & 23.(2) & $1.16(9)$ & $\ldots$ & 0.4 & & $\ldots$ & $\ldots$ & $\ldots$ & $\ldots$ & 0.9 \\
\hline 72 & $8.483(3)$ & $0.208(3)$ & 12.6(2) & 29.(2) & 2.(1) & $3.3(2)$ & $7.7(1)$ & 0.50 & $0.11(4)$ & $0.21(9)$ & $-0.06(9)$ & 1.1 \\
\hline $73^{b}$ & $7.760(2)$ & $<0.462(2)$ & $12.32(6)$ & 89.(1) & 5.(1) & $4.09(6)$ & $8.31(5)$ & 0.58 & $0.13(2)$ & $0.50(9)$ & $0.02(9)$ & 3.3 \\
\hline 75 & $7.335(5)$ & $0.595(5)$ & $15.0(1)$ & 43.(2) & 5.(1) & $2.2(1)$ & 7.1(1) & 0.36 & $0.15(3)$ & $0.32(6)$ & $0.57(6)$ & 1.1 \\
\hline 76 & 7.7(1) & $<0.7(1)$ & $<35$ & $>1.5$ & $\ldots$ & 0.5 & & $\ldots$ & $\ldots$ & $\ldots$ & $\ldots$ & 1.0 \\
\hline 77 & $8.574(2)$ & $0.222(2)$ & $14.3(1)$ & 34.(1) & $\ldots$ & $3.5(1)$ & $9.1(1)$ & 0.55 & $\ldots$ & $\ldots$ & $\ldots$ & 1.4 \\
\hline
\end{tabular}


TABLE 3 - Continued

\begin{tabular}{|c|c|c|c|c|c|c|c|c|c|c|c|c|}
\hline NH3SRC & $\begin{array}{c}V_{L S R} \\
\left(\mathrm{~km} \mathrm{~s}^{-1}\right) \\
(2)\end{array}$ & $\begin{array}{c}\sigma_{v} \\
\left(\mathrm{~km} \mathrm{~s}^{-1}\right) \\
(3)\end{array}$ & $\begin{array}{c}T_{k} \\
(\mathrm{~K}) \\
(4)\end{array}$ & $\begin{array}{c}N_{\mathrm{NH} 3}{ }^{\mathrm{a}} \\
10^{13} \mathrm{~cm}^{-2} \\
(5)\end{array}$ & $\begin{array}{c}N_{\mathrm{CCS}^{\mathrm{a}}} \\
10^{12} \mathrm{~cm}^{-2} \\
(6)\end{array}$ & $\begin{array}{l}\tau_{1} \\
\text { (7) }\end{array}$ & $\begin{array}{l}T_{x}^{\mathrm{a}} \\
(\mathrm{K}) \\
(8)\end{array}$ & $\begin{array}{l}\eta_{f}^{\mathrm{a}} \\
(9)\end{array}$ & $\begin{array}{c}T_{\mathrm{CCS}}{ }^{\mathrm{a}} \\
(\mathrm{K}) \\
(10)\end{array}$ & $\begin{array}{c}\sigma_{\mathrm{CCS}} \\
\left(\mathrm{km} \mathrm{s}^{-1}\right) \\
(11)\end{array}$ & $\begin{array}{c}V_{\text {off }} \\
\left(\mathrm{km} \mathrm{s}^{-1}\right) \\
(12)\end{array}$ & $\begin{array}{l}\widetilde{\chi^{2}} \\
(13)\end{array}$ \\
\hline 78 & $7.12(3)$ & $0.56(3)$ & $13.5(7)$ & $40(1)$ & $\ldots$ & $2.1(5)$ & $5.8(5)$ & 0.28 & $\ldots$ & $\ldots$ & $\ldots$ & 0.8 \\
\hline 79 & $8.26(1)$ & $0.42(1)$ & $15.5(5)$ & $3.4(1)$ & $\ldots$ & \multicolumn{2}{|c|}{$1.91(4)$} & $\ldots$ & $\ldots$ & $\ldots$ & $\ldots$ & 0.8 \\
\hline 80.1 & $7.6(5)$ & $<0.28(2)$ & $11.6(3)$ & 23.(2) & $\ldots$ & $2.26(1)$ & $\ldots$ & 0.30 & $\ldots$ & $\ldots$ & $\ldots$ & 1.6 \\
\hline 80.2 & $8.1(5)$ & $<0.12(2)$ & $10.1(2)$ & 35.(7) & $\ldots$ & $6.33(2)$ & $\ldots$ & 0.22 & $\ldots$ & $\ldots$ & $\ldots$ & 1.6 \\
\hline 81 & $7.487(2)$ & $0.129(2)$ & $11.8(2)$ & 21.(1) & $\ldots$ & $4.2(2)$ & $6.5(1)$ & 0.41 & $\ldots$ & $\ldots$ & $\ldots$ & 0.9 \\
\hline 82.1 & $7.0(2)$ & $0.15(1)$ & $11.9(2)$ & 20.(2) & $\ldots$ & $4.241(3)$ & $\ldots$ & 0.28 & $\ldots$ & $\ldots$ & $\ldots$ & 2.3 \\
\hline 82.2 & $7.5(2)$ & $0.1(1)$ & $13.4(3)$ & 12.(9) & $\ldots$ & $3.710(3)$ & $\ldots$ & 0.15 & $\ldots$ & $\ldots$ & $\ldots$ & 2.3 \\
\hline 83.1 & $7.5(2)$ & $<0.20(7)$ & $11.2(3)$ & 11.(4) & $1.9(2)$ & $1.339(6)$ & $\ldots$ & 0.47 & $0.085(2)$ & $0.34(2)$ & $0.10(2)$ & 3.6 \\
\hline 83.2 & $8.1(1)$ & $<0.10(2)$ & $9.9(3)$ & 31.(8) & $\ldots$ & $6.114(7)$ & $\ldots$ & 0.36 & $\ldots$ & $\ldots$ & $\ldots$ & 3.6 \\
\hline 84 & $7.478(4)$ & $0.146(4)$ & $13.3(4)$ & 15.(2) & $\ldots$ & $4.0(4)$ & $4.8(2)$ & 0.19 & $\ldots$ & $\ldots$ & $\ldots$ & 0.9 \\
\hline 85 & $8.60(7)$ & $<0.55(7)$ & $<22$ & $>0.8$ & $\ldots$ & \multicolumn{2}{|c|}{$0.38(4)$} & $\ldots$ & $\ldots$ & $\ldots$ & $\ldots$ & 1.0 \\
\hline 86 & $7.202(3)$ & $0.136(3)$ & $9.8(4)$ & 22.(3) & $0.7(6)$ & $3.6(3)$ & $5.6(2)$ & 0.40 & $0.16(6)$ & $0.07(3)$ & $-0.02(3)$ & 0.8 \\
\hline 87 & $7.495(1)$ & $0.126(1)$ & $10.4(1)$ & 44.(2) & $1.3(6)$ & $6.6(2)$ & $7.28(7)$ & 0.59 & $0.20(5)$ & $0.11(3)$ & $0.09(3)$ & 0.9 \\
\hline 88.1 & $7.4(2)$ & $0.13(1)$ & $10.3(2)$ & 40.(5) & $\ldots$ & $6.444(3)$ & $\ldots$ & 0.46 & $\ldots$ & $\ldots$ & .. & 2.3 \\
\hline 88.2 & $7.77(7)$ & $0.09(5)$ & $10.5(2)$ & $50(3)$ & $0.2(2)$ & $12.578(3)$ & $\ldots$ & 0.17 & $0.113(4)$ & $0.03(3)$ & $-0.1(1)$ & 2.3 \\
\hline 89 & $8.179(1)$ & $0.125(1)$ & $10.5(1)$ & 39.(2) & $2.1(9)$ & $5.5(2)$ & $7.9(1)$ & 0.66 & $0.18(4)$ & $0.19(5)$ & $0.03(5)$ & 1.2 \\
\hline 90 & $7.88(7)$ & $<0.34(8)$ & $<27$ & $>0.7$ & $\ldots$ & \multicolumn{2}{|c|}{$0.5(1)$} & $\ldots$ & $\ldots$ & $\ldots$ & $\ldots$ & 0.9 \\
\hline 91 & $5.882(1)$ & $0.142(1)$ & $11.0(1)$ & 49.(2) & $\ldots$ & $7.5(2)$ & $6.85(6)$ & 0.50 & $\ldots$ & $\ldots$ & $\ldots$ & 1.1 \\
\hline 93 & $6.022(1)$ & $0.098(1)$ & $10.5(2)$ & 14.(1) & $6.4(4)$ & $3.3(2)$ & $6.1(2)$ & 0.43 & $0.84(3)$ & $0.123(5)$ & $0.019(5)$ & 1.0 \\
\hline 95 & $6.0635(9)$ & $0.1452(8)$ & $10.01(6)$ & 106.(2) & $4.1(5)$ & $13.8(2)$ & $6.75(2)$ & 0.55 & $0.47(4)$ & $0.15(1)$ & $-0.07(1)$ & 1.4 \\
\hline 96 & $7.838(3)$ & $0.152(3)$ & $10.5(3)$ & 19.(2) & $8.2(7)$ & $3.1(3)$ & $5.4(2)$ & 0.34 & $0.95(4)$ & $0.141(7)$ & $0.013(8)$ & 1.0 \\
\hline 97 & $7.724(2)$ & $0.102(2)$ & $9.8(3)$ & $20 .(2)$ & $2.8(4)$ & $4.2(3)$ & $5.5(2)$ & 0.40 & $0.48(4)$ & $0.10(1)$ & $0.03(1)$ & 0.9 \\
\hline 98 & $7.02(3)$ & $<0.34(4)$ & $<20$ & $>1.7$ & $\ldots$ & \multicolumn{2}{|c|}{$1.3(1)$} & $\ldots$ & $\ldots$ & $\ldots$ & $\ldots$ & 1.0 \\
\hline 99 & $6.992(1)$ & $0.182(1)$ & $11.44(7)$ & $49.6(8)$ & $1.5(5)$ & $5.97(9)$ & $7.26(4)$ & 0.52 & $0.09(2)$ & $0.24(6)$ & $-0.07(6)$ & 1.6 \\
\hline 101 & $6.78(5)$ & $<0.22(5)$ & $<23$ & $>6$ & $\ldots$ & 2.(2) & $3.4(6)$ & 0.07 & $\ldots$ & $\ldots$ & $\ldots$ & 0.9 \\
\hline 102 & $6.07(6)$ & $<0.32(8)$ & $<26$ & $>0.8$ & $<10$ & \multicolumn{2}{|c|}{$0.7(1)$} & $\ldots$ & $0.5(1)$ & $0.10(3)$ & $-0.15(7)$ & 1.0 \\
\hline 103 & $6.881(2)$ & $0.243(2)$ & $11.70(9)$ & 62.(1) & 6.(1) & $5.6(1)$ & $7.53(5)$ & 0.53 & $0.40(4)$ & $0.22(3)$ & $0.19(3)$ & 1.3 \\
\hline 104 & $6.420(1)$ & $0.153(1)$ & $10.48(8)$ & 52.(1) & $16.6(5)$ & $7.7(1)$ & $6.07(3)$ & 0.43 & $1.23(2)$ & $0.220(4)$ & $0.041(4)$ & 1.5 \\
\hline 105 & $6.646(2)$ & $0.107(1)$ & $9.6(2)$ & 36.(3) & $2.7(6)$ & $7.7(3)$ & $5.09(6)$ & 0.34 & $0.22(3)$ & $0.22(3)$ & $0.17(3)$ & 1.1 \\
\hline $107^{\mathrm{b}}$ & $6.40(2)$ & $<0.53(2)$ & $<13$ & $>2.7$ & $<6$ & 1.05 & & $\ldots$ & $0.15(2)$ & $0.49(6)$ & $0.05(6)$ & 0.9 \\
\hline 108 & $6.804(1)$ & $0.1283(9)$ & $10.16(9)$ & $72 .(2)$ & $4.7(5)$ & $11.1(2)$ & $6.63(3)$ & 0.52 & $0.51(4)$ & $0.15(1)$ & $-0.02(1)$ & 1.3 \\
\hline 109 & $6.579(6)$ & $0.230(7)$ & $11.2(4)$ & 11.(2) & $4.8(5)$ & $1.4(3)$ & $5.0(4)$ & 0.26 & $0.46(3)$ & $0.16(1)$ & $0.17(1)$ & 0.9 \\
\hline 110 & $6.82(3)$ & $<0.30(3)$ & $<18$ & $>1.1$ & $<3$ & 0.94 & & $\ldots$ & $0.25(5)$ & $0.11(3)$ & $0.18(4)$ & 0.9 \\
\hline 111 & $6.673(1)$ & $0.139(1)$ & $10.1(1)$ & 43.(1) & $5.2(3)$ & $6.2(1)$ & $6.41(5)$ & 0.50 & $0.54(2)$ & $0.161(9)$ & $0.055(9)$ & 1.0 \\
\hline 112 & $6.583(7)$ & $0.257(8)$ & $11.2(5)$ & 11.(3) & $3.8(4)$ & $1.2(3)$ & $5.2(5)$ & 0.29 & $0.40(3)$ & $0.14(1)$ & $0.20(1)$ & 0.9 \\
\hline 113 & $6.570(1)$ & $0.153(1)$ & $10.0(1)$ & 61.(2) & $8.7(4)$ & $7.6(2)$ & $6.68(5)$ & 0.54 & $0.83(3)$ & $0.182(8)$ & $0.100(8)$ & 1.1 \\
\hline 114 & $6.609(1)$ & $0.1894(9)$ & $9.86(6)$ & $106 .(2)$ & $8.7(5)$ & $9.9(1)$ & $7.04(2)$ & 0.60 & $0.83(3)$ & $0.184(7)$ & $0.107(7)$ & 1.8 \\
\hline 115 & $6.574(6)$ & $0.164(6)$ & $10.3(5)$ & 33.(6) & 3.(1) & $4.6(5)$ & $5.8(2)$ & 0.40 & $0.4(1)$ & $0.13(4)$ & $0.01(4)$ & 0.9 \\
\hline 116 & $6.80(4)$ & $<0.57(5)$ & $<16$ & $>17$ & $<4.2$ & $1.9(9)$ & $3.2(2)$ & 0.05 & $0.49(6)$ & $0.08(1)$ & $0.33(5)$ & 0.9 \\
\hline 117 & $6.86(2)$ & $<0.32(3)$ & $<17$ & $>1.3$ & $<4$ & 1.02 & & $\ldots$ & $0.09(3)$ & $0.4(1)$ & $-0.1(1)$ & 0.9 \\
\hline 118 & $6.826(1)$ & $0.1304(9)$ & $9.39(9)$ & $116 .(3)$ & $2.1(6)$ & $15.9(3)$ & $6.29(3)$ & 0.53 & $0.23(4)$ & $0.17(4)$ & $0.08(4)$ & 1.4 \\
\hline 119 & $6.409(1)$ & $0.260(1)$ & $11.52(4)$ & 100.(1) & $12.1(7)$ & $8.10(7)$ & $7.66(2)$ & 0.56 & $0.41(2)$ & $0.43(2)$ & $0.31(2)$ & 2.4 \\
\hline $121^{\mathrm{b}}$ & $6.251(1)$ & $<0.331(1)$ & $12.43(4)$ & 81.(1) & 12.(1) & $5.36(6)$ & $8.17(3)$ & 0.56 & $0.34(2)$ & $0.46(2)$ & $0.24(2)$ & 7.1 \\
\hline 122 & $6.86(4)$ & $<0.33(4)$ & $<22$ & $>0.8$ & $\ldots$ & 0.65 & & $\ldots$ & .. & .. & $\ldots$ & 1.0 \\
\hline 123 & $6.604(1)$ & $0.334(1)$ & $11.71(5)$ & $113 .(2)$ & $11.7(8)$ & $7.21(8)$ & $7.72(3)$ & 0.56 & $0.45(2)$ & $0.36(2)$ & $0.02(2)$ & 2.9 \\
\hline 124 & $6.786(1)$ & $0.121(1)$ & $9.7(1)$ & 61.(2) & $2.0(5)$ & $10.4(3)$ & $5.88(4)$ & 0.45 & $0.28(4)$ & $0.13(2)$ & $0.06(2)$ & 1.2 \\
\hline 125 & $6.48(1)$ & $<0.40(1)$ & $12.0(4)$ & 16.(4) & $4.5(8)$ & $1.5(3)$ & $4.3(2)$ & 0.17 & $0.30(3)$ & $0.21(2)$ & $-0.08(2)$ & 1.0 \\
\hline 126 & $6.790(4)$ & $0.254(5)$ & $11.9(3)$ & 19.(2) & $7.7(5)$ & $1.9(2)$ & $6.4(3)$ & 0.40 & $0.77(4)$ & $0.139(8)$ & $0.034(9)$ & 1.1 \\
\hline $127^{\mathrm{b}}$ & $6.304(6)$ & $<0.260(7)$ & $10.9(3)$ & 22.(3) & $\ldots$ & $2.3(3)$ & $5.2(2)$ & 0.30 & $\ldots$ & ... & $\ldots$ & 1.7 \\
\hline 128 & $7.678(2)$ & $0.079(2)$ & $<10.7$ & $>13$ & $<1.1$ & $5.2(5)$ & $4.2(1)$ & 0.22 & $0.27(4)$ & $0.07(1)$ & $0.06(1)$ & 0.9 \\
\hline 129 & $7.59(3)$ & $<0.32(4)$ & $<20$ & $>1.4$ & $\ldots$ & 1.2( & & $\ldots$ & $\ldots$ & $\ldots$ & $\ldots$ & 1.0 \\
\hline 132 & $7.06(1)$ & $<0.17(1)$ & $<15$ & $>5$ & $<3$ & $1.6(9)$ & $3.8(5)$ & 0.13 & $0.24(5)$ & $0.12(3)$ & $0.05(3)$ & 0.9 \\
\hline 139 & $5.29(4)$ & $0.43(4)$ & 24.(2) & $0.64(8)$ & $\ldots$ & 0.40 & & $\ldots$ & $\ldots$ & $\ldots$ & $\ldots$ & 0.9 \\
\hline 141 & $8.19(2)$ & $<0.16(2)$ & $<16$ & $>6$ & $<6$ & 2.(1) & $5 .(1)$ & 0.29 & $0.4(1)$ & $0.14(4)$ & $0.02(4)$ & 0.9 \\
\hline 142 & $8.475(3)$ & $0.113(3)$ & $12.4(4)$ & 10.(2) & $3.3(8)$ & $3.0(5)$ & $5.1(3)$ & 0.24 & $0.35(5)$ & $0.12(2)$ & $0.07(2)$ & 1.0 \\
\hline 144 & $8.24(2)$ & $0.21(2)$ & 20.(2) & $0.57(9)$ & $\ldots$ & 0.73 & & $\ldots$ & .. & .. & .. & 0.9 \\
\hline 145 & $9.431(3)$ & $0.094(2)$ & $9.5(4)$ & 43.(6) & 2.(1) & $8.6(7)$ & $6.1(2)$ & 0.50 & $0.4(1)$ & $0.10(3)$ & $0.07(3)$ & 0.9 \\
\hline 146 & $8.18(1)$ & $<0.18(1)$ & 17.(1) & 5.(3) & $\ldots$ & $1.4(9)$ & $3.7(5)$ & 0.07 & $\ldots$ & $\ldots$ & $\ldots$ & 1.0 \\
\hline 147 & $9.432(1)$ & $0.0980(9)$ & $9.6(1)$ & 52.(2) & $1.5(5)$ & $9.6(3)$ & $6.54(6)$ & 0.55 & $0.28(5)$ & $0.10(2)$ & $0.02(2)$ & 0.9 \\
\hline 148 & $9.43(2)$ & $<0.15(2)$ & $<18$ & $>10$ & $\ldots$ & 4.(2) & $4.1(5)$ & 0.14 & ... & $\ldots$ & $\ldots$ & 0.9 \\
\hline 150 & $8.54(5)$ & $<0.47(6)$ & $<20$ & $>7$ & $\ldots$ & 1.(1) & $3.1(4)$ & 0.04 & $\ldots$ & $\ldots$ & $\ldots$ & 1.0 \\
\hline 151 & $8.64(2)$ & $<0.28(3)$ & $<17$ & $>0.9$ & $<3$ & 0.84 & & $\ldots$ & $0.20(4)$ & $0.14(3)$ & $0.06(4)$ & 0.9 \\
\hline 152 & $8.82(1)$ & $0.14(1)$ & 14.(2) & $6 .(4)$ & 4.(2) & 2.(1) & $5 .(1)$ & 0.17 & $0.5(1)$ & $0.10(3)$ & $0.06(3)$ & 0.9 \\
\hline 153 & $8.64(5)$ & $<0.20(5)$ & $<23$ & $>9$ & $\ldots$ & 3.(3) & $3.3(3)$ & 0.05 & $\ldots$ & ... & .. & 0.9 \\
\hline 154 & $8.71(5)$ & $<0.39(6)$ & 17.(3) & $1.4(3)$ & $\ldots$ & 0.9 & & $\ldots$ & $\ldots$ & $\ldots$ & $\ldots$ & 1.0 \\
\hline 156 & $8.55(1)$ & $0.35(2)$ & $13.5(6)$ & 19.(5) & $\ldots$ & $1.7(4)$ & $6.0(6)$ & 0.31 & $\ldots$ & $\ldots$ & $\ldots$ & 0.9 \\
\hline 157.1 & $8.3(2)$ & $0.16(3)$ & $12.9(6)$ & 12.(2) & $\ldots$ & $2.150(9)$ & $\ldots$ & 0.15 & $\ldots$ & $\ldots$ & $\ldots$ & 1.9 \\
\hline 157.2 & $8.7(6)$ & $0.14(3)$ & $12.3(5)$ & 15.(3) & $1.4(5)$ & $2.95(1)$ & $\ldots$ & 0.33 & $0.156(3)$ & $0.12(3)$ & $0.07(3)$ & 1.9 \\
\hline 158 & $9.011(4)$ & $0.134(4)$ & $14.8(4)$ & $2.3(1)$ & $2.3(5)$ & 3.8 & & $\ldots$ & $0.41(6)$ & $0.058(9)$ & $0.06(1)$ & 1.3 \\
\hline 159 & $8.709(2)$ & $0.180(2)$ & $11.7(1)$ & 28.(1) & $1.5(6)$ & $3.5(1)$ & $7.2(1)$ & 0.50 & $0.16(4)$ & $0.14(4)$ & $0.12(4)$ & 1.1 \\
\hline 160 & $8.637(4)$ & $0.195(4)$ & $11.7(3)$ & 36.(3) & ... & $3.5(3)$ & $8.6(3)$ & 0.65 & ... & $\ldots$ & $\ldots$ & 0.9 \\
\hline 161 & $8.984(5)$ & $0.224(5)$ & $12.9(3)$ & 40.(3) & $\ldots$ & $4.3(3)$ & $7.6(2)$ & 0.48 & $\ldots$ & $\ldots$ & $\ldots$ & 0.9 \\
\hline 162 & $8.742(9)$ & $0.22(1)$ & $14.2(6)$ & 13.(4) & $\ldots$ & $1.7(5)$ & 7.(1) & 0.39 & $\ldots$ & $\ldots$ & $\ldots$ & 0.9 \\
\hline 163 & $8.269(3)$ & $0.179(3)$ & $12.9(2)$ & 20.(1) & $1.9(6)$ & $3.8(2)$ & $5.3(1)$ & 0.25 & $0.18(4)$ & $0.14(3)$ & $0.02(3)$ & 1.0 \\
\hline
\end{tabular}


TABLE 3 - Continued

\begin{tabular}{|c|c|c|c|c|c|c|c|c|c|c|c|c|}
\hline $\begin{array}{c}\text { NH3SRC } \\
\text { (1) }\end{array}$ & $\begin{array}{c}V_{L S R} \\
\left(\mathrm{~km} \mathrm{~s}^{-1}\right) \\
(2)\end{array}$ & $\begin{array}{c}\sigma_{v} \\
\left(\mathrm{~km} \mathrm{~s}^{-1}\right) \\
(3)\end{array}$ & $\begin{array}{c}T_{k} \\
(\mathrm{~K}) \\
(4)\end{array}$ & $\begin{array}{c}N_{\mathrm{NH} 3^{\mathrm{a}}} \\
10^{13} \mathrm{~cm}^{-2} \\
(5)\end{array}$ & $\begin{array}{c}N_{\mathrm{CCS}^{\mathrm{a}}} \\
10^{12} \mathrm{~cm}^{-2} \\
(6)\end{array}$ & (7) & $\begin{array}{l}T_{x}^{\mathrm{a}} \\
(\mathrm{K}) \\
(8)\end{array}$ & $\begin{array}{l}\eta_{f}^{\mathrm{a}} \\
(9)\end{array}$ & $\begin{array}{c}T_{\mathrm{CCS}}{ }^{\mathrm{a}} \\
(\mathrm{K}) \\
(10)\end{array}$ & $\begin{array}{c}\sigma_{\mathrm{CCS}} \\
\left(\mathrm{km} \mathrm{s}^{-1}\right) \\
(11)\end{array}$ & $\begin{array}{c}V_{o f f} \\
\left(\mathrm{~km} \mathrm{~s}^{-1}\right) \\
(12)\end{array}$ & (13) \\
\hline 164 & $9.003(9)$ & $0.27(1)$ & $13.0(5)$ & 17.(4) & $\ldots$ & $1.5(4)$ & $7.6(9)$ & 0.48 & $\ldots$ & $\ldots$ & $\ldots$ & 0.8 \\
\hline 165 & $8.471(2)$ & $0.184(2)$ & 11.1(1) & 41.(2) & $\ldots$ & $5.5(2)$ & $6.21(6)$ & 0.41 & $\ldots$ & $\ldots$ & $\ldots$ & 3.2 \\
\hline 166 & $8.28(3)$ & $0.38(4)$ & 26.(2) & $0.70(9)$ & $\ldots$ & \multicolumn{2}{|c|}{$0.51(4)$} & $\ldots$ & $\ldots$ & $\ldots$ & $\ldots$ & 1.0 \\
\hline 167 & $8.28(4)$ & $<0.54(5)$ & $<21$ & $>1.1$ & $\ldots$ & \multicolumn{2}{|c|}{$0.56(4)$} & $\ldots$ & $\ldots$ & $\ldots$ & $\ldots$ & 1.0 \\
\hline 168 & $8.14(2)$ & $0.22(3)$ & 11.(2) & 7.(7) & $\ldots$ & 1.(1.) & 5.(3) & 0.45 & $\ldots$ & $\ldots$ & $\ldots$ & 1.0 \\
\hline 169 & $8.473(4)$ & $0.154(4)$ & $10.9(4)$ & 32.(4) & $\ldots$ & $4.6(4)$ & $6.5(2)$ & 0.46 & $\cdots$ & $\ldots$ & $\ldots$ & 0.9 \\
\hline 170 & $8.983(5)$ & $0.137(5)$ & $10.6(6)$ & 21.(5) & $\cdots$ & $3.1(5)$ & $6.9(5)$ & 0.53 & $\cdots$ & $\cdots$ & $\ldots$ & 0.9 \\
\hline 171 & $7.920(5)$ & $0.160(5)$ & $12.9(4)$ & 8.(2) & $\cdots$ & $1.6(4)$ & $5.7(5)$ & 0.29 & $\cdots$ & $\cdots$ & $\cdots$ & 0.9 \\
\hline 173 & $9.134(4)$ & $0.153(4)$ & $10.5(4)$ & 33.(4) & $\ldots$ & $3.9(4)$ & $7.8(3)$ & 0.65 & $\cdots$ & $\ldots$ & $\ldots$ & 0.9 \\
\hline 174 & $7.59(2)$ & $<0.37(2)$ & $14.7(9)$ & $2.6(2)$ & $\ldots$ & \multicolumn{2}{|c|}{$1.56(7)$} & $\ldots$ & $\ldots$ & $\ldots$ & $\ldots$ & 0.9 \\
\hline 176 & $9.968(4)$ & $0.166(4)$ & $10.8(3)$ & 17.(3) & $\ldots$ & $2.5(3)$ & $5.7(3)$ & 0.37 & $\ldots$ & $\ldots$ & $\ldots$ & 1.1 \\
\hline 178 & $9.89(2)$ & $0.34(3)$ & 16.(1) & $2.7(3)$ & $\ldots$ & \multicolumn{2}{|c|}{$1.9(1)$} & & $\ldots$ & $\ldots$ & $\ldots$ & 1.0 \\
\hline 180 & $8.954(5)$ & $0.121(4)$ & $10.8(6)$ & 21.(4) & $\ldots$ & $4.3(6)$ & $5.8(3)$ & 0.37 & $\ldots$ & $\ldots$ & $\ldots$ & 0.9 \\
\hline 181 & $9.096(4)$ & $0.138(4)$ & $10.9(5)$ & 8.(3) & $0.8(4)$ & $1.3(4)$ & $6.2(9)$ & 0.43 & $0.08(3)$ & $0.16(8)$ & $0.05(8)$ & 1.0 \\
\hline 182 & $9.14(3)$ & $<0.19(4)$ & $<22$ & $>0.5$ & $\ldots$ & \multicolumn{2}{|c|}{$0.7(1)$} & 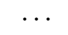 & $\ldots$ & $\ldots$ & $\ldots$ & 1.0 \\
\hline 183 & $9.992(4)$ & $0.109(3)$ & $10.7(5)$ & 23.(4) & $\ldots$ & $5.0(6)$ & $6.0(3)$ & 0.41 & $\ldots$ & $\ldots$ & $\ldots$ & 0.9 \\
\hline 188 & $10.151(2)$ & $0.107(2)$ & $10.0(3)$ & 15.(2) & $\ldots$ & $2.6(3)$ & $6.6(3)$ & 0.53 & $\ldots$ & $\ldots$ & $\ldots$ & 1.0 \\
\hline 189 & $10.370(6)$ & $0.182(5)$ & 10.1(4) & 44.(5) & 5.(2) & $5.1(4)$ & $6.1(2)$ & 0.46 & $0.24(6)$ & $0.3(1)$ & $-0.1(1)$ & 0.9 \\
\hline 190 & $10.130(3)$ & $0.119(3)$ & $10.1(4)$ & 14.(2) & $9.3(9)$ & $3.0(4)$ & $5.1(2)$ & 0.32 & $0.98(3)$ & $0.161(6)$ & $-0.005(7)$ & 1.0 \\
\hline 191 & $10.131(3)$ & $0.127(3)$ & $10.0(4)$ & 26.(3) & $7.0(6)$ & $5.2(5)$ & $4.8(1)$ & 0.29 & $0.77(4)$ & $0.16(1)$ & $-0.05(1)$ & 0.9 \\
\hline 192 & $10.243(4)$ & $0.164(4)$ & $11.7(3)$ & 41.(4) & 3.(2) & $6.0(4)$ & $6.8(2)$ & 0.45 & $0.3(1)$ & $0.13(5)$ & $0.08(5)$ & 0.9 \\
\hline
\end{tabular}

NotE. - (1) Source Number from Tablen (2) Source velocity with respect to the LSR. (3) Velocity dispersion of the $\mathrm{NH}_{3}$ (4) Kinetic temperature. (5) Column Density of $\mathrm{NH}_{3}$ assuming $\eta_{f}=1$. (6) Column Density of $\mathrm{C}_{2} \mathrm{~S}$. (7) Total opacity in the $\mathrm{NH}_{3}(1,1)$ line. (8) Excitation Temperature for the $\mathrm{NH}_{3}$. For lines-of-sight on which the opacity and excitation temperature cannot be separately determined, the product $\tau_{1}\left(T_{x}-T_{b g}\right)$ is quoted, spanning both columns. (9) Filling fraction for the case where $T_{K}=T_{x}$. (10) Peak temperature of $\mathrm{C}_{2} \mathrm{~S}$. (11) Velocity dispersion of the of $\mathrm{C}_{2} \mathrm{~S}$ line. (12) Velocity offset of the $\mathrm{C}_{2} \mathrm{~S}$ line with respect to the $\mathrm{NH}_{3}$ complex. (13) reduced $\chi^{2}$ for the fit (since data are not completely independent, this is only a goodness-of-fit parameter).

a This property is affected by the overall amplitude calibration which is subject to an additional $\sim 5 \%$ uncertainty.

$\mathrm{b}$ The spectrum shows evidence for multiple components in one of the spectral lines that cannot be resolved uniquely by multicomponent fitting. 